\title{
AN ANNOTATED CHECKLIST OF TASMANIAN MOSSES
}

\author{
by P.J. Dalton, R.D. Seppelt and A.M. Buchanan
}

An annotated checklist of the Tasmanian mosses is presented to clarify the occurrence of taxa within the state. Sorne recently collected species, for which there are no published records, have been included. Doubtful records and excluded species are listed separately. The Tasmanian moss flora as recognised here includes 361 species.

Key Words: mosses, Tasmania.

In BANKS, M.R. et al. (Eds), 1991 (31:iii): ASPECTS OF TASMANIAN BOTANY - A TRIBUTE TO WINIFRED CURTIS. Roy. Soc. Tasm. Hobart: 15-32. https://doi.org/10.26749/rstpp.124.2.15

\section{INTRODUCTION}

Tasmanian mosses received considerable attention during the early botanical exploration of the antipodes. One of the earliest accounts was given by Wilson (1859), who provided a series of descriptions of the then-known species, accompanied by coloured illustrations, as Part III of J.D. Hooker's Botany of the Antarctic Voyage. Although there have been a number of papers since that time, two significant compilations were published about the turn of the century. The first was by Bastow (1887) which included an illustrated key. Rodway (1913-14) presented a further moss flora with descriptive keys for many genera. Although published as three separate papers in Pap. Proc. R. Soc. Tasm. (1912: 3-24, 87-138 and 1913: 177-263), Rodway's work was reissued as a single volume Tasmanian Bryophyta. Vol. I. Mosses, in 1914. For clarity we refer to this single volume publication. This was the last account which dealt exclusively with Tasmanian mosses. They have since been included in wider accounts of the moss flora of Australia. Scott \& Stone (1976) provided a much needed comprehensive treatment of the mosses of the southern Australian region including Tasmania. Streimann \& Curnow (1989) published a catalogue of the mosses of Australia and its offshore islands and antarctic territory. In that work, the occurrence of taxa in the Australian states is based primarily on literature records, some of which are in need of evaluation.

The impetus for the present checklist arose from a need for a working document for the Australasian Bryological Workshop held in Hobart in December 1988. It is intended to provide a basis of what is known to occur in the Tasmanian moss flora by evaluation of literature records combined with re-examination of critical collections. There are Tasmanian records in the literature of a number of taxa for which there are no known collections available. It is possible that some others may be the result of misidentification or misinterpretation of the original reference. Furthermore, in recent years previously unrecorded species have been found as well as several new taxa described.

We have assigned genera to amilies following Crosby \& Magill (1981), except where otherwise indicated in the case of more recent publications. The arrangement of families, genera and species is in alphabetic order for ease of access. Taxa known to occur in Tasmania and its neighbouring islands only are listed; those for subantarctic Macquarie Island (politically part of Tasmania) are not treated and have been presented elsewhere (Seppelt 1981).

This Tasmanian checklist complements those for the vascular flora (Buchanan et al. 1989), hepatics (Ratkowsky 1987) and lichens (Kantvilas 1989).

\section{ANNOTATED CHECKLIST OF TASMANIAN MOSSES BY FAMILY IN ALPHABETICAL ORDER}

All entries are presented in a format where accepted names are in bold type and unaccepted names or synonyms are in plain text. The synonyms given are by no means exhaustive, but the list attempts to include all names that have been used in Tasmania. Infraspecific ranks are not treated comprehensively. An asterisk indicates species or varieties which are endemic in Tasmania. Annotations include an early reference to occurrence in Tasmania, followed by general comments and taxonomic notes where appropriate. Doubtful or excluded records are listed separately in plain text.

\section{AMBLYSTEGIACEAE}

Acrocladium auriculatum (Mont.) Mitt. = Acrocladium chlamydophyllum

Acrocladium chlamydophyllum (Hook.f. et Wils.) C.Mucll. et Broth. Bastow (1887) as Hypnum chlamydophyllum; Rodway (1914). Sometimes referred to Lembophyllaceae on the basis of a double peristome.

Acrocladium (uspidatum (Hedw.) Lindb. = Calliergonella cuspidata 
Acrocladium politum Hook.f. et Wils. = Catagonium politum (Plagiotheciaceae)

Amblystegium serpens (Hedw.) B.S.G. Rodway (1915) as A. austro-serpens hom.illeg.; Sainsbury (1956b).

Calliergonella cuspidata (Hedw.) Loeske. Beever (1988) separates Acrocladium sensu stricto from Acrocladium (Calliergonella) on leaf shape, Kanda (1977) considered Calliergonella as anomalous in Amblystegiaceae and resembling members of Hypnaceae.

Campylium decussatum (Hook.f. et Wils.) Broth. = Cratoneuropsis relaxa

Campylium molle Broth. in Rodw. = Cratone uropsis relaxa

Campylium polygamum (B.S.G.) C.Jens. Ratkowsky \& Ratkowsky (1983).

Campylium relaxum (Hook.f, et Wils.) Broth. = Cratoneuropsis relaxa

Cratoneuropsis relaxa (Hook.f. et Wils.) Fleisch. Rodway (1914); Sainsbury (1956b).

Drepanocladus aduncus (Hedw.) Warnst. Rodway (1914); Sainsbury (1956b).

Drepanocladus brachiatus (Mitt.) Dix. Rodway (1914); Sainsbury (1956b).

Drepanocladus fluitans (Hedw.) Warnst. Bastow (1887) as Hypnum fluitans; Rodway (1914).

Drepanocladus sendtneri (Schimp.) Wamst. Rodway (1915); Sainsbury $(1956 b)=D$. aduncus .

Drepanocladus uncinatus (Hedw.) Warnst. Scott \& Stone (1976). Rare, no collections in HO.

Platyhypnidium austrinum (Hook.f. et Wils.) Fleisch. = Eurhynchium austrinum (Brachytheciaceae)

Scorpidium scorpioides (Hedw.) Limpr. Jarman et al. (1988) as Scorpidium aff. scorpioides. Formerly considered to be a Northern Hemisphere plant, one specimen from southwestern Tasmania.

\section{ANDREAEACEAE}

The only two costate species of Andreaea in Tasmania, A. nitida and $A$. subulata, are easily distinguished. The remaining ecostate species are the subject of a major revision currently being undertaken by Dr B. Murray, University of Alaska, Fairbanks.

Andreaea acuminata Mitt. in Hook.f. et Wils. = Andreaea acutifolia var. acuminata

Andreaea acutifolia Hook.f. et Wils.

var, acuminata (Mitt. in Hook.f. et Wils.) Vitt. Mitten (1860); Rodway (1914) as A. acuminata.

var, acutifolia.

Andreaea amblyophylla C.Muell. ex Broth. = Andreaea mutabilis

Andreaea asperula Mitt. Mitten (1860); Rodway (1916).

Andreaea erubescens* C.Muell. (? = Andreaea mutabilis) var, nigrita * C.Muell. (? = Andreaea mutabilis)

Andreaea eximia* C.Muell. Rodway (1914) but no collections in $\mathrm{HO}$.

Andreaea julicaulis C.Muell. (? = Andreaea mutabilis)

Andreaea montana Mitt. = Andreaea mutabilis

Andreaea mutabilis Hook.f. et Wils. Mitten (1860); Bastow (1887); Rodway (1914) as A. montana.

Andreaea nitida Hook.f. et Wils. Mitten (1882); Rodway (1914).

Andreaea nupestris Hedw. (Australian material) = Andreaea mutabilis. Vitt (1980a).
Andreaea subulata Harv, ex Hook. Mitten (1882); Rodway (1914) as $A$. subulatissima.

Andreaea subulatissima C.Muell, = Andreaea subulata

Andreaea tasmanica Rodw. Rodway (1916).

Andreaea tenera C.Muell. = Andreaea mutabilis

\section{AULACOMNIACEAE}

Aulacomnium palustre (Hedw.) Schwaegr. Wilson (1859); Sainsbury (1955d). Formerly considered to be a Northern Hemisphere moss, rare in Tasmania, no collections in $\mathrm{HO}$.

Leptotheca gaudichaudii Schwaegr. var. gaudichaudii Mitten (1882); Rodway (1914).

var. wattsii* (Card.) Churchill et Buck. Churchill \& Buck (1982); there are no collections in HO.

Leptotheca wattsii Card. = Leptotheca gaudichaudii var. wattsii

\section{BARTRAMIACEAE}

Bartramia erecta (Hampe) Broth. = Bartramia hampei

Bartramia fragilis Mitt. = Bartramia ithyphylla

Bartramia halleriana $\mathrm{Hedw}$. Rodway (1914) as B. norvegica. var, brachydonta Kab.

Bartramia hampeana C.Muell. Ratkowsky \& Ratkowsky (1982) but see Scott and Stone (1976), Catcheside (1980).

Bartramia hampei (Mitt.) Catches. Rodway (1914) as $B$. erecta; Catcheside (1980). Rare, no material in $\mathrm{HO}$.

Bartramia ithyphylla Brid. Bastow (1887) as B. fragilis; Rodway (1914).

Bartramia norvegica Lindb. nom. illeg. = Bartramia halleriana

Bartramia papillata Hook.f. et Wils. = Bartramia ithyphylla

Bartramia stricta Brid. Rodway (1914) as B. strictifolia.

Bartramia strictifolia Tayl. = Bartramia stricta

Bartramidula hampe Mitt. = Bartramia hampei

Bartramidula pusilla (Hook.f. et Wils.) Par. Mitten (1882); Rodway (1914).

Bartramidula weymouthii Broth. in Weymouth nom. nud. = Bartramidula pusilla

Breutelia affinis (Hook.) Mitt. Mitten (1882); Rodway (1914).

Breutelia comosa (Mitt.) Mitt. = Breutelia pendula

Breutelia crassa (Hook.f. et Wils.) Jaeg. = Breutelia elongata Breutelia divaricata (Mitt.) Mitt. = Breutelia pendula

Breutelia elongata (Hook.f. et Wils.) Mitt. Mitten (1882); Rodway (1914) as B. crassa.

Breutelia pendula (Sm.) Mitt. Mitten (1882); Rodway (1914).

Conostomum australe Sw. = Conostomum pentastichum

Conostomum pentastichum (Brid.) Lindb. Mitten (1882) and Rodway (1914) as C. australe.

Conostomum pusillum Hook.f. et Wils. Mitten (1882); Rodway (1914).

Philonotis appressa (Hook.f. et Wils.) Mitt. = Philonotis scabrifolia

Philonotis australis Mitt. = Conostomum pentastichum

Philonotis fertilis (Mitt.) Mitt. = Philonotis tenuis

Philonotis rigens Broth. = ?Philonotis tenuis Sainsbury (1955e).

Philonotis scabrifolia (Hook.f. et Wils.) Braithw. Mitten (1882) as P. appressa; Rodway (1914).

Philonotis tenuis (Tayl.) Reichdt. Mitten (1882); Rodway (1914); Sainsbury (1955e). 


\section{BRACHYTHECIACEAE}

Brachythecium albicans (Hedw.) B.S.G. = Chamberlainia albicans Robinson (1962).

Brachythecium paradoxum (Hook.f. et Wils.) Jaeg. Mitten (1882); Bastow (1887); Rodway (1914) as Hypnum paradoxum.

Brachythecium plumosum (Hedw.) B.S.G. Record based on one collection in $\mathrm{HO}$ (det. Norris).

Brachythecium rutabulum (Hedw.) B.S.G. Mitten (1882); Bastow (1887) and Rodway (1914) as Hypnum rutabulum.

Brachythecium salebrosum (Web. et Mohr) B.S.G. = Chamberlainia salebrosa. Robinson (1962).

Chamberlainia albicans (Hedw.) H.Robinson. Scott \& Stone (1976) as Bracythecium albicans.

Chamberlainia salebrosa (Web. et Mohr) H.Robinson. Mitten (1882) as Brachythecium salebrosum; Rodway (1914) as Hypnum salebrosum.

Eurhynchium asperipes (Mitt.) Dix. Bastow (1887) as Hypnum asperipes; Rodway (1914) as Hypnum remotifolium.

Eurhynchium austrinum (Hook.f. et Wils.) Jaeg. Bastow (1887); Rodway (1914) as Hypnum austrinum.

Eurhynchium cucullatum (Mitt.) Stone et Scott. Weymouth (1896) as Hypnum convolutifolium.

Eurhynchium muriculatum (Hook.f. et Wils.) Jaeg. = Rhynchostegiella muriculata

Eurhynchium praelongum (Hedw.) B.S.G. = Kindbergia praelonga Ochyra (1982).

Kindbergia praelonga (Hedw.) Ochyra. Sainsbury (1956b) as Eurhynchium praelongum

Rhynchostegiella muriculata (Hook.f. et Wils.) Broth. Mitten (1882) as Rhynchostegium muriculatum; Bastow (1887); Rodway (1914) as Hypnum muriculatum.

Rhynchostegium aristatum Jaeg. = Rhynchostegium laxatum Rhynchostegium laxatum (Mitt.) Par. Bastow (1887); Rodway (1914) as Hypnum aristatum.

Rhynchostegium tenuifolium (Hedw.) Reichdt. Bastow (1887) as Hypnum collatum; Rodway (1914) as Hypnum tenuifolium.

Rhynchostegium muriculatum (Hook.f. et Wils.) Reichdt. = Rhynchostegiella muriculata

\section{BRUCHIACEAE}

Bruchia exigua (Hook.f. et Wils.) C.Muell. = Eccremidium exiguum (Ditrichaceae)

Bruchia minuta Mitt. in Hook.f. = Eccremidium minutum (Ditrichaceae)

\section{BRYACEAE}

The genus Bryum is currently under revision as part of the Flora of Australia project.

Apalodium australe (Hook.f. et Wils.) Mitt. = Orthodontium lineare

Apalodium lineare $($ Tayl.) Mitt. = Orthodontium pallens

Brachymenium lanceolatum* Hook.f. et Wils. Mitten (1882). However, there are no collections in HO to support this early record.

Brachymenium preissianum (Hampe) Jaeg. Weymouth (1896); Rodway (1914).

Bryum argenteum Hedw. Mitten (1882); Rodway (1914).
Bryum argillicola Broth. $=$ Bryum pachytheca

Bryum bicolor Dicks. = Bryum dichotomum

Bryum billardieri Schwaegr. Mitten (1882); Rodway (1914). var. billardieri

Bryum bimum (Schreb.) Turn. = Bryum pseudotriquetrum

Bryum blandum Hook.f. et Wils. Mitten (1882); Rodway

(1914).

var, crinitum Wils.

var. luridum Wils.

Bryum breviramulosum (Hampe) Hampe $=$ Bryum billardieri

Bryum caespiticium Hedw. Bastow (1887).

Bryum campylothecium Tayl. Rodway (1914).

Bryum capillare Hedw. Mitten (1882); Rodway (1914).

Bryum chrysoneuron C.Muell. Mitten (1882); Rodway (1914) but see Scott \& Stone (1976).

Bryum clavatum (Schimp.) C.Muell. Weymouth (1896) as $B$. erythrocarpoides.

Bryum crassum Hook.f. et Wils. Mitten (1882); Rodway (1914).

Bryum creberrimum Tayl. Weymouth (1896).

Bryum cupulatum C.Muell.$=$ Bryum pachytheca

Bryum curvicollum Mitt. = Bryum clavatum

Bryum dichotomum Hedw. Rodway (1914).

Bryum erythrocarpoides C.Muell. et Hampe = Bryum clavatum

Bryum gambierense C.Muell $=$ Bryum pachytheca

Bryum intermedium (Brid.) Blani. = ?Bryum caespiticium. Sainsbury (1955d).

Bryum laevigatulum Broth. = ?Bryum clavatum . Sainsbury (1955d).

Bryum laevigatum Hook.f. et Wils. Mitten (1882); Rodway (1914).

Bryum leptothecium Tayl $=$ Bryum billardieri var. billardieri

Bryum microerythrocarpum C.Muell. et Kindb. = Bryum subapiculatum

Bryummicrorhodon C.Muell. $=$ ?Bryumbillardieri. Sainsbury (1955d).

Bryum microsporum Broth. = Bryum capillare

Bryum ovicarpum Broth. = Bryum pachytheca

Bryum pachytheca C.Muell. Weymouth (1903) as $B$. ovicarpum.

var. crassinerve Watts et Whitel.

var. inflatum Wils.

Bryum pseudotriquetrum (Hedw.) Gaertn., Meyer et Scherb. Rodway (1914) as B. bimum; Sainsbury (1955d).

Bryum pyrothecium C.Muell. et Hampe = Bryum capillare . Sainsbury (1955d).

Bryum rufescens Hook.f. et Wils. $=$ Bryum billardieri

Bryum sauteri B.S.G. Duncan \& Dalton (1982).

Bryum subapiculatum Hampe. Ratkowsky and Ratkowsky (1982) as B. microerythrocarpum.

Bryum subcupulatum C.Muell. ex Rodw. = Bryum dichotomum

Bryum sullivani C.Muell. $=$ Bryum clavatum

Bryum tasmanicum Hampe $=$ Bryum creberrimum. Sainsbury (1955d).

Bryum torquescens Bruch Bastow (1887); Rodway (1914). Bryum truncorum Brid.= Bryum billardieri var. billardieri

Leptobryum pyriforme (Hedw.) Wils. Bastow (1887); Rodway (1914).

Leptobryum sericeum* Kindb. Bastow (1887). Scott \& Stone 
(1976) consider likely to be synonymous with $L$. pyriforme. However, there are no collections in $\mathrm{HO}$.

Leptostomu $n$ flexipile C.Muell = Leptostomum inclinans Leptostomu $n$ gracile $\mathrm{R} . \mathrm{Br},=$ Leptostomum inclinans

Leptostomuin inclinans R.Br. Mitten (1882); Bastow (1887); Rodway (1914).

Mielichhoferia bryoides (Harv.) Wijk et Marg. Rodway (1914) as $M$.echloni.

Mielichhoferia ecklonii Hornsch. = Mielichhoferia bryoides

Mniobryum tasmanicum Broth. = Pohlia wahlenbergii

Mniobryum wahlenbergii (Web. et Mohr) Jenn. - Pohlia wahlenbergii

Orthodontium australe Hook.f. et Wils. = Orthodontium lineare

ssp. robustiusculum (C.Muell.) Meijer = Orthodontium lineare

Orthodontium lanceolatum Mitt. = Orthodontium lineare ssp. sulcatum

Orthodontium lineare Schwaegr. Mitten (1882) as Apalodium australe; Rodway (1914).

ssp. sulcatum (Hook.f. et Wils.) Meijer. Meijer (1952).

Orthodontium pallens (Hook.f. et Wils.) Broth. Sainsbury (1955d); Scott \& Stone (1976).

Orthodontium robustiusculum C.Muell. = Orthodontium lineare

Pohlia albicans Lindb. = Pohlia wahlenbergii

Pohlia annotina (Hedw.) Lindb. Recently collected from highland areas (A.J. Fife 8781, 8821).

Pohlia cruda (Hedw.) Lindb. Rodway (1914).

Pohlia nutans (Hedw.) Lindb. Rodway (1914).

Pohlia ochii Vitt. Recently collected from Mt Wellington (A.J. Fife 8820):

Pohlia tasmanica (Broth.) Dix. = Pohlia wahlenbergii

Pohlia wahlenbergii (Web. et Mohr) Andrews in Grout. Weymouth (1894) as Mniobryum tasmanicum.

\section{BUXBAUMIACEAE}

Buxbaumia aphylla Hedw. Scott \& Stone (1976). Very rare, existing for a short period almost solely as a sporopbyte.

Buxbaumia tasmanica* Mitt. Mitten (1860); Rodway (1914). Very rare, existing for a short period almost solely as a sporophyte.

\section{CALOMNIACEAE}

Calomnion complanatum (Hook.f. et Wils.) Lindb. A new record which is known from only one recent collection by I.G. Stone, northwestern Tasmania, epiphyte.

\section{CRYPHAEACEAE}

Cryphaea consimilis Mont. $=$ Cryphaea tenella

Cryphaea dilatata Hook.f. et Wils. = Cyptodon dilatatus. See Doubtful and Excluded Records.

Cryphae a parvula sensu Rodway $1914=$ Cryphaea tenella . Sainsbury (1955f).

Cryphaea tasmanica Mitt. in Hook.f. et Wils. Mitten (1882); Rodway (1914) as Dendrocryphaea tasmanica.

Cryphaea tenella (Schwaegr.) Hornsch. ex C.Muell. Sainsbury (1955a).

Dendrocryphaea tasmanica (Mitt.) Broth. = Cryphaea tasmanica

\section{DAWSONIACEAE}

Dawsonia longifolia (B.S.G.) Zanten. Mitten(1882); Rodway (1914) as D. superba.

var. longifolia

Dawsonia longiseta Hampe. Scott \& Stone (1976). Uncommon, known from only a few collections.

Dawsonia polytrichoides R.Br. Scott \& Stone (1976). Uncommon, known from only a few collections.

Dawsonia superba Grev. = Dawsonia longifolia var. longifolia. Scott \& Stone (1979) argued for the conservation of the more familiar name $D$. superba

\section{DICNEMONACEAE}

Eucamptodon inflatus (Hook.f.et Wils.) Mitt. = Pulchrinodus inflatus (Pterobryaceae)

\section{DICRANACEAE}

Anisothecium crispum Lindb. $=$ Anisothecium schreberianum

Anisothecium schreberianum (Hedw.) Dix. Sainsbury (1955b); Scott \& Stone (1976) suggested would be better placed in Dicranella.

Campylopodium euphorocladum (C.Muell.) Besch. = Campylopodium medium

Campylopodium flexipes (Mitt.) Broth. = Campylopodium medium

Campylopodium lineare (Mitt.) Dix. Rodway (1914) as Cynodontium tasmanicum; Sainsbury (1955b).

Campylopodium medium (Dub.) Giese et Frahm. Rodway (1914) as C.flexipes; Sainsbury (1955b).

Campylopus acuminatus Mitt.

var. kirkii (Mitt.) Frahm. Weymouth (1903) as C. kirkii; Frahm (1987).

Campylopus angustilimbatus Bartram = Campylopus australis

Campylopus arboricola Card. et Dix. Scott \& Stone (1976). Campylopus australis Catches. et Frahm. Catcheside \& Frahm (1985) do not record for Tasmania. However, collections are held in HO det. D. Catcheside.

Campylopus bicolor (Hornsch. ex C.Muell.) Wils. Mitten (1882); Rodway (1914), but Frahm (1987) did not record for Tasmania.

var. ericeticola (C.Muell.) Dix.

Campylopus brunneus* (C.Muell.) Par. Watts \& Whitelegge (1902). No material in HO, see Frahm (1987).

Campylopus clavatus (R.Br.) Wils. Mitten (1882); Bastow (1887) as C. insititius; Rodway (1914).

Campylopus denticuspis Broth. = Campylopus acuminatus var. kirkii

Campylopus flavonigritus Dus. Weymouth (1903) as C. subappressifolius; Catcheside \& Frahm (1985); Frahm (1987) treats as $C$. chilensis. Rare, no collections in HO.

Campylopus flindersii Catches. et Frahm. Catcheside \& Frahm (1985) do not record for Tasmania. Pers. comm. with Catcheside gives a collection from northern Tasmania.

Campylopus insititius Hook.f. et Wils. = Campylopus clavatus. Frahm (1987).

Campylopus introflexus (Hedw.) Brid. Bastow (1887); Rodway (1914).

ssp. pudicus (Hornsch. ex C.Muell.) Dix. 
mpylopus kirkii Mitt. =Campylopus acuminatus var. kirkii ampylopus pallidus Hook.f. et Wils. = Campylopus pyriformis

Campylopus pyriformis (Schultz) Brid. Mitten (1882) as C. torquatus; Scott \& Stone (1976) as C.pallidus; Frahm (1987).

Campylopus rodwayi Broth. in Rodw. = Campylopus clavatus

Campylopus subappressifolius Broth. et Geh. = Campylopus flavonigritus

Campylopus tasmanicus Par. = Campylopus introflexus

Campylopus torquatus Mitt. = Campylopus pyriformis

Cynodontium tasmanicum Broth. et Rodw. = Campylopodium lineare

Dicnemoloma pallidum (Hook.) Wijk et Marg. Bastow (1887) as Dicranum sieberianum; Rodway (1914) as Sclerodontium pallidum; Sainsbury (1955b) as D. sieberianum.

Dicnemoloma sieberianum (Hornsch.) Broth. = Dicnemoloma pallidum

Dicranella cardotii (R.Br.ter.) Dix. Scott \& Stone (1976).

Dicranella dietrichiae (C.Muell.) Jaeg. Ratkowsky and Ratkowsky (1983). Uncommon, known from only a few collections.

Dicranella jamesonii (Mitt.) Broth. Sainsbury (1955b); Scott \& Stone (1976).

Dicranella schreberiana (Hedw.) Hilf. = Anisothecium schreberianum

Dicranodontium tapes* (C.Muell.) Par. Watts \& Whitelegge (1902); Sainsbury (1955b) gave as Tasmanian endemic but not referred to by Rodway (1914). No collections in $\mathrm{HO}$.

Dicranoloma angustiflorum* Dix. Scott \& Stone (1976) do not know this species. No material in $\mathrm{HO}$.

Dicranoloma angustifolium* (Hook.f. et Wils.) Watts et Whitel. Watts \& Whitelegge (1906). No material in HO.

Dicranolomaaustrinum (Mitt.) Watts et Whitel. Mitten(1882) as Dicranum austrinum; Watts \& Whitelegge (1906). No material in $\mathrm{HO}$.

Dicranoloma billarderi (Brid.) Par. Mitten (1882); Rodway (1914) as Dicranum billardieri.

*var. angustinerve (Rodw.) Sainsb. Rodway (1914).

var. billarderi

Dicranoloma burchardtii* (Par.) Par. Weymouth (1896) as Dicranum rigens. Rare, no material in $\mathrm{HO}$.

Dicranoloma diaphanoneurum (Hampe) Par. = ?Dicranoloma austrinum. Catcheside (1980).

Dicranoloma dicarpum (Nees) Par. Mitten (1882) as Dicranum dicarpum; Rodway (1914) as Dicranum polysetum.

Dicranoloma eucamptodontoides (Broth. et Geh.) Par. Weymouth (1896); Rodway (1914) as Dicranum elicamptodontoides. Previously treated as endemic in Tasmania, but now (A.J. Fife, pers. comm.) known also from Fiordland, New Zealand. Occurs in wet buttongrass sedgeland in southwestern Tasmania.

Dicranoloma menziesii (Tayl.) Par. Bastow (1887); Rodway (1914) as Dicranum menziesii; Sainsbury (1955b).

Dicranoloma nelsonï* (C.Muell.) Par. Watts \& Whitelegge (1906). Rare, no material in HO.
Dicranoloma perichaetiale* Sainsb. Sainsbury ( 1953a). Rare, known from only one collection, subalpine.

Dicranoloma platycaulon (C.Muell.) Dix. Scott \& Stone (1976).

Dicranoloma robustum (Hook.f.et Wils.) Par. Bastow (1887); Rodway (1914) as Dicranum robustum; Sainsbury (1955b). Scott \& Stone (1976) include in D. billardieri.

var. setosum (Hook.f. et Wils.) Sainsb. Bastow (1887); Rodway (1914) as Dicranum setosum; Sainsbury (1955b).

Dicranoloma setosum Hook.f. et Wils. = Dicranoloma robustum var, setosum

Dicranoloma subsetosum (C.Muell.) Par. = Dicranoloma robustum var. setosum

Dicranoloma trichopodum (Mitt.) Broth. Weymouth \& Rodway (1922) as Dicranum trichopodum; Sainsbury (1955b).

Dicranoweisa antarctica (C.Muell.) Kindb. Sainsbury (1955b); Willis (1955b).

Dicranoweisia microcarpa (Hook.f. et Wils.) Par. Mitten (1882) as Dicranum microcarpum; Rodway (1914) as Weissia microcarpa; Sainsbury (1955b).

Dicranum austrinum Mitt. $=$ Dicranoloma austrinum

Dicranum billardieri Brid. $=$ Dicranoloma billardieri

Dicranum dicarpum Nees = Dicranoloma dicarpum

Dicranum eucamptodontoides Broth. et Geh. = Dicranoloma eucamptodontoides

Dicranum integerrimum Broth. et Geh. = Dicranoloma billardieri

Dicranum menziesii Tayl. $=$ Dicranoloma menziesii

Dicranum microcarpum (Hook.f. et Wils.) Mitt. = Dicranoweisia microcarpa

Dicranum polysetum Hampe = Dicranoloma dicarpum

Dicranum pungens Hook.f. et Wils. = Dicranoloma robustum

Dicranum rigens Burchard et Broth. ex C.Muell. = Dicranoloma burchardtii

Dicranum robustum Hook.f. et Wils. = Dicranoloma robustum

Dicranum setosum Hook.f. et Wils. = Dicranoloma robustum var. setosum

Dicranum sieberianum Hornsch, = Dicnemoloma pallidum

Dicranum trichopodum Mitt. $=$ Dicranoloma trichopodum

Holomitrium perichaetiale (Hook.) Brid. Mitten (1882); Rodway (1914).

Leucoloma sieberianum (Hornsch.) Jaeg. = Dicnemoloma pallidum

Sclerodontium pallidum $($ Hook.) Schwaegr. = Dicnemoloma pallidum

Trematodon flexipes Mitt. in Hook.f. Mitten (1882); Weymouth \& Rodway (1922); Sainsbury (1955b).

Trematodon mackayi (R.Br.ter.) Broth. Weymouth \& Rodway (1922).

\section{DITRICHACEAE}

Ceratodon purpureus (Hedw.) Brid. Bastow (1887); Rodway (1914).

Cheilothela chilensis (Mont.) Broth. = Chrysoblastella chilensis. Reimers (1926); Buck (1981).

Chrysoblastella chilensis (Mont.) Reim. Ratkowsky (1980) 
as Cheilothela chilensis. Widespread in alpine and subalpine areas in Tasmania.

Distichium capillaceum (Hedw.) B.S.G. Bastow (1887); Rodway (1914); Scott \& Stone (1976). Known in Tasmania only from the Gordon River limestone.

Ditrichum australe (Mitt.) Mitt. = Ditrichum strictum

Ditrichum brevirostre (R.Br.ter.) Broth. Record based on one collection in $\mathrm{HO}$ determined by $\mathrm{R}$. Seppelt.

Ditrichum cylindricarpum (C.Muell.) F.Muell.Mitten (1882); Rodway (1914) as D. elongatum.

Ditrichum difficile (Dub.) Fleisch. Weymouth (1896); Rodway (1914) as D. flavipes

Ditrichum elongatum (Hook.f. et Wils.) Mitt. = Ditrichum cylindricarpum

Ditrichum flavipes C.Muell. ex Rodw. = Ditrichum difficile Ditrichum flexifolium (Mitt.) Hampe = Ditrichum difficile

Ditrichum oldfieldii (Mitt.) Mitt. = Ditrichum difficile

Ditrichum punctulatum Mitt. Weymouth \& Rodway (1922).

Ditrichum strictiusculum (C.Muell.) Par. = Ditrichum cylindricarpum

Ditrichum strictum (Hook.f. et Wils.) Hampe. Mitten (1882) as $D$. australe. Sainsbury (1955b) did not see any Tasmanian material attributable to this species.

Ditrichum subcapillaceum* (C.Muell.) Watts et Whitel. Watts \& Whitelegge (1902). Scott \& Stone (1976)=Distichium capillaceum.

Eccremidium exiguum (Hook.f. et Wils.) Wils. Record based on one old collection (W.H.Archer) determined by I.G. Stone.

Eccremidium minutum (Mitt.) Stone et Scott . Mitten (1882); Rodway (1914) as Bruchia minuta.

Eccremidium pulchellum (Hook.f. et Wils.) C.Muell. Scott \& Stone (1976); Ratkowsky \& Ratkowsky (1982).

Leptotrichum australe Mitt. = Ditrichum strictum

Pleuridium arnoldii (R.Br.ter.) Par. Record based on a collection in HO determined by D.H. Norris.

Pleuridium gracilentum (Mitt.) Mitt. = Pleuridium nervosum

Pleuridium lampropyxis* (C.Muell.) Par. Watts \& Whitelegge (1902).

Pleuridium nervosum (Hook.) Mitt. Watts \& Whitelegge (1902); Sainsbury (1955b).

Pleuridium ienellum (Mitt.) Mitt. = ?Pleuridium nervosum

\section{ECHINODIACEAE}

Echinodium hispidum (Hook.f. et Wils.) Reichdt. Bastow (1887) as Hypnum hispidum; Rodway (1914).

\section{ENCALYPTACEAE}

Encalypta novaevalesiae Hampe. Hampe (1872). However, Scott \& Stone (1976) record only for Victoria and New South Wales.

Encalypta vulgaris Hedw. Bastow (1887); Rodway (1914) as E. australis.

var. mutica Brid. Paris (1895).

Encalypta australis Mitt. = Encalypta vulgaris

\section{ENTODONTACEAE}

Entodon tasmanicus* Mitt. Mitten (1882) described this for Tasmania but there are no collections in HO.

Pseudoscleropodium purum (Hedw.) Fleisch. Ratkowsky \& Ratkowsky (1983).
EPHEMERACEAE

Ephemerum cristatum (Hook.f. et Wils.) C.Muell. Mitten (1882); Rodway (1914).

\section{EPHEMEROPSIDACEAE}

Ephemeropsis trentepohlioides (Renn.) Sainsb. Willis (1953). Fomerly considered endemic in New Zealand. A rare epiphyte on dead twigs in humid forest.

\section{ERPODIACEAE}

Leptangium see Gigaspermaceae

\section{FABRONIACEAE}

Fabronia australis Hook. Scott \& Stone (1976). Uncommon in wet Eucalyptus forest.

Ischyrodon lepturus (Tayl.) Schelpe. Harris \& Ratkowsky (1982). A rare coastal moss, known in Tasmania from only two localities.

\section{FISSIDENT ACEAE}

Fissidens adianthoides Hedw. Mitten (1882); Bastow (1887); Sainsbury (1955d).

Fissidens asplenioides Hedw. Bastow (1887); Sainsbury (1955d).

Fissidens brevifolius Hook.f. et Wils. = Fissidens taylorii

Fissidens dealbatus Hook.f. et Wils. Mitten (1882); Bastow (1887); Rodway (1914).

Fissidens elamellosus C. Muell. et Hampe $=$ Fissidens taylorii

Fissidens fontanus (Pyl.) Steud. Catcheside (1980) but there are no collections in HO.

Fissidens incurvus Starke ex Roehl. Mitten (1882); Bastow (1887) as F. incurvis. Only one collection (W.H. Archer) held in $\mathrm{HO}$.

Fissidens integerrimus* Mitt. Mitten(1882); Rodway (1914); Sainsbury (1955d).

Fissidens leptocladus C.Mue!1. ex Rodw. Weymouth (1903); Rodway (1914).

Fissidens oblongifolius Hook.f. et Wils. Mitten (1882); Bastow (1887); Rodway (1914).

Fissidens pallidus Hook.f. et Wils. Mitten (1882); Bastow (1887); Rodway (1914).

Fissidens pungens C.Muell. et Hampe. Bastow (1887); Rodway (1914).

Fissidens rigidulus Hook.f. et Wils. Mitten (1882); Bastow (1887); Rodway (1914).

ssp. rigidulus

Fissidens semilimbatus C.Muell. et Hampe $=$ Fissidens taylorii

Fissidens strictus Hook.f. et Wils. Mitten (1882); Bastow (1887); Rodway (1914).

Fissidens taylorii C.Muell. Mitten (1882); Bastow (1887); Rodway (1914).

var. floribundus (Wils.) Wijk et Marg.

Fissidens tenellus Hook.f. et Wils. Mitten (1882); Bastow (1887); Rodway (1914).

Fissidens tortuosus* Geh. et Hampe. Watts \& Whitelegge (1902) is the only record for Tasmania. No locality is given and no collection in $\mathrm{HO}$.

Fissidens vittatus Hook.f. et Wils. Mitten (1882); Bastow (1887); Rodway (1914). 
Fissidens whiteleg gii C.Muell, ex Rodw.=Fissidens pallidus

\section{FUNARIACEAE}

Entosthodon gracilis Hook.f. et Wils. = Entosthodon subnudus var. gracilis

Entosthodon laxus (Hook.f. et Wils.) Mitt. = Physcomitrium laxum

Entosthodon productus Mitt. = Funaria producta

Entosthodon subnudus (Tayl.) Fife var. gracilis (Hook.f. et Wils.) Fife. Bastow (1887) as E. gracilis; Rodway (1914) as Funaria gracile.

Funaria apophysata (Tayl.) Broth. Bastow (1887) as Physcomitrium apophysatum; Rodway (1914).

Funaria calvescens Schwaegr. = Funaria hygrometrica var. calvescens

Funaria crispula Hook.f. et Wils. = Funaria glabra

Funaria cuspidata Hook.f. et Wils. = Entosthodon subnudus var. gracilis

Funaria glabra Tayl. Mitten (1882); Bastow (1887); Rodway (1914).

Funaria gracilis (Hook.f. et Wils.) Broth. = Entosthodon subnudus var. gracilis

Funaria hygrometrica Hedw. Bastow (1887); Rodway (1914). Watts \& Whitelegge (1906) referred to this species in Southern Hemisphere as $F$. hygrometrica var. sphaerocarpa.

var. calvescens (Schwaegr.) Mont. Watts \& Whitelegge (1906).

var. sphaerocarpa (C.Muell.) Watts. Watts \& Whitelegge (1906); Weymouth (1894) as $F$. sphaerocarpa.

Funaria producta (Mitt.) Broth. Mitten (1882) as Entosthodon productus; Rodway (1914).

Physcomitrium conicum Mitt. in Hook. Mitten (1882); Bastow (1887); Rodway (1914).

Physcomitrium laxum (Hook.f. et Wils.) C.Muell. Rodway (1914) considered this to be possibly a form of $P$. conicum.

Physcomitrium apophysatum (Tayl.) Wils. = Funaria apophysata

\section{GIGASPERMACEAE}

Gigaspermum repens (Hook,) Lindb. Bastow (1887) as Leptangium repens, Rodway (1914). Uncommon. Restricted to the low rainfall region of southeastern Tasmania.

Leptangium repens Mitt. = Gigaspermum repens

Leptangium tumidium Mitt. = ?Gigaspermum repens. Sainsbury (1955e).

\section{GRIMMIACEAE}

Grimmia apocarpa Hedw. = Schistidium apocarpum

Grimmia austrofunalis C.Muell. Record based on two collections by D.H. Norris (27623 \& 28171). Previously known only from highland areas of southeastern mainland Australia.

Grimmia basaltica Mitt. = Grimmia pulvinata

Grimmia cygnicolla Tayl. = Grimmia pulvinata

Grimmia laevigata (Brid.) Brid. Bastow (1887) as G. leucophaea; Rodway (1914) as G. leiocarpa.

Grimmia leiocarpa Tayl. = Grimmia laevigata

Grimmia leucophaea Grev. = Grimmia laevigata
Grimmia pulvinata (Hedw.) Sm. Bastow (1887); Sainsbury (1955c).

var. africana (Hedw.) Hook.f. et Wils.

Grimmia pygmaea C.Muell. Watts \& Whitelegge (1902).

Grimmia stenophylla* C.Muell. Watts \& Whitelegge (1902), but no collections at $\mathrm{HO}$.

Grimmia tasmanica* C.Muell. Watts \& Whitelegge (1902). Sainsbury $(1955 \mathrm{c})$ placed in synonomy with $G$. pulvinata.

Grimmia trichophylla Grev. Bastow (1887); Rodway (1914).

Racomitrium crispulum (Hook.f. et Wils.) Hook.f. et Wils. Bastow (1887); Rodway (1914). For recent taxonomic treatment of varieties see Lawton (1973).

var. rupestre (Hook.f. et Wils.) Dix. = Racomitrium rupestre

var. tasmanicum (Hampe) Lawton

Racomitrium heterostichum (Hedw.) Brid. Bastow (1887); Rodway (1914).

var. affine (Web. et Mohr) Amann

var. obtusum (Brid.) Delogn.

Racomitrium lanuginosum (Hedw.) Brid. ssp. lanuginosum Mant. Vitt \& Marsh (1988).

Racomitrium lanuginosum (Hedw.) Brid. var, pruinosum Hook.f. et Wils. $=\boldsymbol{R}$. pruinosum

Racomitrium pruinosum (Wils.) C.Muell. Bastow (1887) as R. lanuginosum var. pruinosum; Vitt \& Marsh (1988).

Racomitrium ptychophyllum (Mitt.) Mitt. Ratkowsky \& Ratkowsky (1983).

Racomitrium rupestre (Hook.f. et Wils.) Hook.f. et Wils. Bastow (1887); Rodway (1914).

Racomitrium symphyodon Jaeg. = Racomitrium crispuium

Schistidium apocarpum (Hedw.) B.S.G. Bastow (1887); Rodway (1914) as G. apocarpa.

\section{HEDWIGIACEAE}

Braunia humboldtii (Hook.) Hook.f. = Rhacocarpus purpurascens

Harrisonia australis (Hook.f. et Wils.) Hampe= Rhacocarpus purpurascens

Hedwigia albicans Lindb. = Hedwigia ciliata

Hedwigia ciliata (Hedw.) Ehrh. ex P.Beauv. Bastow (1887); Rodway (1914) as H. albicans.

Hedwigia imberbis (Sm.) Spruc. = Hedwigidium integrifolium

Hedwigia integrifolia P.Beauv. = Hedwigidium integrifolium

Hedwigia microcyathea (C.Muell.) F.Muell. = Hedwigia ciliata

Hedwigidium imberbe (Sm.) B.S.G. = Hedwigidium integrifolium

Hedwigidium integrifolium (P.Beauv.) Dix. in Jens. Bastow (1887) as Hedwigia imberbis; Rodway (1914) as Hedwigidium imberbis.

Rhacocarpus australis (Hook.f. et Wils.) Par. = Rhacocarpus purpurascens

Rhacocarpus humboldtii (Hook.) Lindb. = Rhacocarpus purpurascens

Rhacocarpus purpurascens (Brid.) Par. Bastow (1887) as Braunia humboldtil; Rodway (1914) as Rhacocarpus australis. 


\section{HOOKERIACEAE}

Achrophyllum dentatum (Hook.f. et Wils.) Vitt et Crosby. Bastow(1887) as Hookeria denticulata; Rodway (1914) as Pterygophyllum denticulatum.

Calyptrochaeta apiculata (Hook.f. et Wils.) Vitt. Bastow (1887) as Hookeria apiculata; Rodway (1914) as Eriopus apiculatus.

Calyptrochaeta flexicollis (Mitt. in Hook.f.) Vitt. Sainsbury (1956a) as Eriopus flexicollis.

Daltonia angustifolia Dozy et Molk. (Tasmanian material) $=$ Daltonia splachnoides. Sainsbury (1956a); Scott \& Stone (1976).

Daltonia pusilla Hook.f. et Wils. (Tasmanian material) = Daltonia splachnoides. Sainsbury (1956a); Scott \& Stone (1976).

Daltonia splachnoides (Sm.) Hook. et Tayl. Bastow (1887); Rodway (1914) as Daltonia pusilla.

Distichophyllum assimile Broth. Fleischer (1922); Welch (1970). There are no collections held in HO.

Distichophyllum beccarii (C.Muell. ex Hampe et Geh.) Par. Paris (1895); Welch (1970). There are no collections held in $\mathrm{HO}$.

Distichophyllum complanatum (Hampe) Mitt. Brotherus (1907); Welch (1970). There are no collections held in HO.

Distichophyllum crispulum (Hook.f. et Wils.) Mitt. Mitten (1882); Rodway (1914); Sainsbury (1956a). However, no collections are held at $\mathrm{HO}$.

Distichophyllum kraussei (Lor.) Mitt. A new record based on a collection by A.J. Fife (8967) in 1988.

Distichophyllum levieri(Geh.) Broth. Brotherus(1907); Welch (1970). There are no collections held in HO.

Distichophyllum microcarpum (Hedw.) Mitt. Mitten (1882); Rodway (1914).

Distichophyllum pulchellum (Hampe) Mitt. Mitten (1882); Rodway (1914).

Distichophyllum rotundifolium (Hook.f. et Wils.) C.Muell. et Broth. Rodway 1914; Sainsbury 1956a

Eriopus apiculatus (Hook.f. et Wils.) Mitt. = Calyptrochaeta apiculata. Vitt (1979).

Eriopus brownii Dix. Willis (1957).

Eriopus flexicollis (Mitt.) Jaeg. $=$ Calyptrochaeta flexicollis . Vitt (1979).

Hookeria amblyophylla Hook.f. et Wils. = Distichophyllum pulchellum

Hookeria apiculata Hook.f. et Wils. = Calyptrochaeta apiculaia

Hookeria denticulata Hook.f. et Wils. = Achrophyllum dentatum

Hookeria nigella Hook.f. et Wils. $=$ Achrophyllum dentatum

Hookeria obscurum Mitt. = Pterygophyllum obscurum

Hookeria pennata (Labill.) Sm. nom. illeg. = Cyathophorum bulbosum (Hypopterygiaceae)

Hookeria tenella Hook.f. et Wils. = Sauloma tenella

Mniadelphus amblyophyllus (Hook.f. et Wils.) Jaeg. = Distichophyllum pulchellum

Mniadelphus microcarpus (Hedw.) C.Muell. = Distichophyllum microcarpum

Mniadelphus pulchellus Hampe = Distichophyllum pulchellum

Mniadelphus rotundifolius (Hook.f. et Wils.) C.Muell. = Distichophyllum rotundifolium
Mniadelphus sinuosus (Hook.f. et Wils.) Jaeg. = Distichophyllum pulchellum

Pterygophyllum dentatum (Hook.f. et Wils.) Dix. = Achrophyllum dentatum

Pterygophyllum denticulatum Mitt. = Achrophyllum dentatum

Pterygophyllum flaccidissimum* Broth. Brotherus (1909); Scott \& Stone (1976) also list for Tasmania. However, there are no collections in HO.

Pterygophyllum hookeri Jaeg. = Pterygophyllum obscurum

Pterygophyllum levieri Geh. $=$ Distichophyllum levieri

Pterygophyllum microcarpum (Hedw.) Brid. = Distichophyllum microcarpum

Pterygophyllum nigellum (Hook.f. et Wils.) Mitt. = Achrophyllum dentatum

Pterygophyllum obscurum Mitt. Mitten 1860; Bastow (1887) as Hookeria obscurum

Sauloma tenella (Hook.f. et Wils.) Mitt. Bastow (1887) as Hookeria tenella; Rodway (1914).

\section{HYPNACEAE}

Hypnum aciculare Labill. = Ptychomnion aciculare (Ptychomniaceae)

Hypnum amoenum Hedw. = Rhaphidorrhynchium amoenum (Sematophyllaceae)

Hypnum aristatum Hook.f. et Wils. = Rhynchostegium laxatum (Brachytheciaceae)

Hypnum asperipes Mitt. = Eurhynchium asperipes (Brachytheciaceae)

Hypnum austrinum Hook.f. et Wils. = Eurhynchium ausirinum (Brachytheciaceae)

Hypnum callidioides C.Muell. = Rhaphidorrhynchium callidioides (Sematophyllaceae)

Hypnum cerviculatum Hook.f. et Wils. = Sematophyllum leucocytus (Sematophyllaceae)

Hypnum chlamydophyllum Hook.f. et Wils. = Acrocladium chlamydophyllum (Amblystegiaceae)

Hypnum chrysogaster C.Muell. Rodway (1914) as Stereodon chrysogaster

Hypnum clandestinum Hook.f. et Wils. = Lembophyllum divulsum (Lembophyllaceae)

Hypnum cochlearifolium Schwaegr, = Weymouthia cochlearifolia (Meteoriaceae)

Hypnum collatum Hook.f. et Wils. = Rhynchostegium tenuifolium (Brachytheciaceae)

Hypnum comosum Labill. = Hypnodendron comosum (Hypnodendraceae)

Hypnum contiguum Hook.f. et Wils. = Sematophyllum contiguum (Sematophyllaceae)

Hypnum convolutifolium Hampe = Eurhynchium cucullatum (Brachytheciaceae)

Hypnum crinitum Hook.f. et Wils. = Wijkia extenuata (Sematophyllaceae)

Hypnum cupressiforme Hedw. Bastow (1887); Sainsbury (1956b).

var. cupressiforme

var. filiforme Brid. Sainsbury (1956b); Scott \& Stone (1976).

var. lacunosum Brid. Sainsbury (1956b).

var. tectorum Brid. Scott \& Stone (1976).

Hypnum divulsum Hook.f. et Wils. = Lembophyllum divulsum (Lembophyllaceae) 
Hypnum fluitans Hedw. = Drepanocladus fluitans (Amblystegiaceae)

Hypnum hispidum Hook.f. et Wils. = Echinodium hispidum (Echinodiaceae)

Hypnum jolliffii Hook.f. $=$ Rhaphidorrhynchium jolliffii (Sematophyllaceae)

Hypnum moorei Broth. et Geh. = Eurhynchium austrinum (Brachytheciaceae)

Hypnum muriculatum Hook.f. et Wils. = Rhynchostegiella muriculata (Brachytheciaceae)

Hypnum nelsonii* (Broth.) Par. Weymouth (1896); Rodway (1914) as Stereodon nelsonii. Known only from old collections, restricted to southeastern Tasmania.

Hypnum paradoxum Hook.f. et Wils. = Brachythecium paradoxum (Brachytheciaceae)

Hypnum remotifolium Hook.f. et Wils. = Eurhynchium asperipes (Brachytheciaceae)

Hypnum rutabulum Hedw. = Brachythecium rutabulum (Brachytheciaceae)

Hypnum tenuifolium Hedw. $=$ Rhynchostegium tenuifolium (Brachytheciaceae)

Hypnum walterianum (Hampe) Jaeg. = Hypnum cupressiforme

Isopterygium acuminatum* Boswell. Weymouth (1894); Rodway (1914); Sainsbury (1956b).

Isopterygium albescens (Hook.) Jaeg. Miller et al. (1978) list for Tasmania; however, Scott \& Stone (1976) record only from the Australian mainland.

Isopterygium limatum (Hook.f. et Wils.) Broth. Rodway (1914); Sainsbury (1956b). Uncommon and localised in wet sites.

Stereodon chrysogaster (C. Muell.) Mitt. = Hypnum chrysogaster

Stereodon cupressiformis (Hedw.) Brid. ex Mitt. = Hypnum cupressiforme

Stereodon flagelliramens Broth. = Wijkia extenuata (Sematophyllaceae)

Stereodon jolliffii (Hook.f.) Mitt. = Rhaphidorrhynchium jolliffii (Sematophyllaceae)

Stereodon nelsonii Broth. $=$ Hypnum nelsonii (Broth.) Par.

\section{HYPNODENDRACEAE}

Hypnodendron archeri (Mitt.) Jaeg. = Hypnodendron spininervium ssp. archeri

Hypnodendron arcuatum (Hedw.) Lindb. ex Mitt. = Hypnodendron vitiense ssp. australe

Hypnodendron comosum (Labill.) Mitt.

var. comosum. Bastow (1887) as Isothecium comosum; Touw (1971).

var. sieberi (C.Muell.) Touw. Bastow (1887) as lsothecium sieberi; Touw (1971).

Hypnodendron spininervium (Hook.) Jaeg.

ssp. archeri (Mitt.) Touw. Bastow (1887) as Isothecium archeri; Rodway (1914) as Hypnodendronarcheri; Touw (1971).

Hypnodendron vitiense Mitt.

ssp. australe Touw. Bastow (1887) as Isothecium spininervium; Touw (1971).

Mniodendron comosum (Labill.) Lindb. ex Par. = Hypnodendron comosum var. comosum

Mniodendron sieberi (C.Muell.) Jaeg. ex Par. = Hypnodendron comosum var. sieberi

\section{HYPOPTERYGIACEAE}

Cyathophorum bulbosum (Hedw.) C.Muell. Bastow (1887) as C. pennatum; Rodway (1914).

var. minus (Hook.f. et Wils.) Par. Weymouth (1894); Rodway (1914) as C. densirete.

Cyathophorum densirete Broth. $=$ Cyathophorum bulbosum var. minus

Cyathophorum pennatum (Labill.) Brid. = Cyathophorum bulbosum

Hypopterygium ciliatum (Hedw.) Brid. = Catharomnion ciliatum. See Doubtful and Excluded Records.

Hypopterygium didictyon C.Muell. Mitten (1882) as H. Novae Zealandiae; Rodway (1914) as $H$. novae-zelandiae; Bastow (1887) as H. glaucum. However, Scott \& Stone (1976) consider that this species is not satisfactorily distinguished from $H$. rotulatum.

Hypopterygium glaucum Sull. = Hypopterygium didictyon

Hypopterygium novaese elandiae C.Muell, = Hypopterygium didictyon

Hypopterygium pallens (Hook.f. et Wils.) Mitt. = Lopidium concinnum

Hypopterygium rotulatum (Hedw.) Brid. Scott \& Stone (1976).

Hypopterygium setigerum Wils. = Hypopterygium commutatum

Hypopterygium smithianum Hook.f. et Wils. = Hypopterygium didictyon

Hypopterygium tasmanicum C.Muell. ex Kindb. = Hypopterygium didictyon

Lopidium concinnum (Hook.) Wils. Bastow (1887); Rodway (1914) as Lopidium pallens.

Lopidium pallens Hook.f. et Wils. = Lopiaium concinnum

\section{LEMBOPHYLLACEAE}

Camptochaete arbuscula (Sm.) Reichdt. Bastow (1887) as Isothecium arbuscula; Rodway (1914).

var. deflexa (Wils.) Dix. Rodway (1914) as Camptochaete deflexa.

Camptochaete deflexa (Wils.) Jaeg. = Camptochaete arbuscula var. deflexa

Camptochaete gracilis (Hook.f. et Wils.) Par. Rodway (1914) as C. gracile.

Camptochaete ramulosa (Mitt.) Jaeg. = Camptochaete arbuscula. Scott \& Stone (1976).

Camptochaete tasmanica Broth. = Camptochaete gracilis. Rodway (1914); Sainsbury (1955f) considers as a form of $C$. gracilis.

Camptochaete vaga (Homsch. ex C. Muell.) Broth. Mitten (1882) as Porotrichum vagum. Sainsbury (1955f) inferred occurrence in Tasmania. Scott \& Stone (1976) did not indicate as occurring in Tasmania.

Isothecium angustatum (Mitt.) Hook.f. = Camptochaete angustata. See Doubtful and Excluded Records.

Isothecium arbuscula $(\mathrm{Sm}$. $)$ Brid. = Camptochaete arbuscula

Isothecium archer $i$ Mitt. $=$ Hypnodendron spininervium $\mathrm{ssp}$. archeri (Hypnodendraceae)

Isothecium colensoi Hook. f. et Wils. = Hypnodendron colensoi. See Doubtful and Excluded Records.

Isothecium comosum (Labill.) Brid. = Hypnodendron comosum var. comosum (Hypnodendraceae)

Isothecium fruticosom (Mitt.) Bastow = Camptochaete fruticosa. See Doubtful and Excluded Records. 
Isothecium pumilum Hook.f. et Wils. = Thamnobryum pumilum

Isothecium ramulosum Mitt. = Camptochaete arbuscula

Isothecium sieberi $(\mathrm{C}$. Muell.) Wils. = Hypnodendron comosum var. sieberi (Hypnodendraceae)

Isothecium spininervium (Hook.) Wils. = Hypnodendron vitiense ssp. australe (Hypnodendraceae)

Lembophyllum clandestimum (Hook.f. et Wils.) Lindb. = Lembophyllum divulsum var. clandestinum

Lembophyllum cochlearifolium (Schwaegr.) Lindb. = Weymouthia cochlearifolia (Meteoriaceae)

Lembophyllum divulsum (Hook.f. et Wils.) Lindb. Rodway (1914).

var. clandestinum (Hook.f. et Wils.) Wijk et Marg. Rodway (1914) treated at specific rank. Sainsbury (1955f) and Scott \& Stone (1976) treat only as a variety.

\section{LEPYRODONTACEAE}

Lepyrodon lagurus (Hook.) Mitt. Bastow (1887) as Leucodon lagurus; Rodway (1914).

\section{LEUCOBRYACEAE}

Leucobryum candidum (P.Beauv.) Wils. Bastow (1887); Rodway (1914). Sainsbury (1955b) considered varieties in Tasmanian material to be referable to the typical plant. var. majus (Schwaegr.) Jaeg. ex Dix.

var. pentastichum (Dozy et Molk.) Dix.

Leucobryum confusum Ther. $=$ ? Leucobryum candidum

\section{LEUCODONTACEAE}

Leucodon lagurus Hook. = Lepyrodon lagurus (Lepyrodontaceae)

\section{METEORIACEAE}

Barbella nitens (Hook.f. et Wils.) Nog. Rodway (1914) as Papillaria intricata; Sainsbury (1955f) as Papillaria nitens.

Meteorium billardieri (Hampe) Mitt. = Weymouthia cochlearifolia var. billardieri

Meteorium cerinum Hook.f. et Wils. = Papillaria flavolimbata

Meteorium filipendulum Hook.f. et Wils. = Papillaria flexicaulis

Meteorium molle Wils. $=$ Weymouthia mollis

Papillaria crocea (Hampe) Jaeg. Rodway (1914) as P. kermadecensis; Sainsbury (1955f).

Papillaria filipendula (Hook.f. et Wils.) Jaeg. = Papillaria flexicaulis

Papillaria flavolimbata (C.Muell. et Hampe) Jaeg. Bastow (1887) as Meteorium cerinum; Rodway (1914); Sainsbury (1955f).

Papillaria flexicaulis (Wils.) Jaeg. Bastow (1887) as Meteorium filipendulum; Rodway (1914) as Papillaria filipendula; Sainsbury (1955f).

Papillaria intricata (Mitt.) C.Muell. et Broth. = Barbella nitens. Noguchi (1985).

Papillaria kermadecensis (C.Muell.) Jaeg. = Papillaria crocea

Papillaria nitens (Hook.f. et Wils.) Sainsb. = Barbella nitens Noguchi (1985).
Weymouthia cochlearifolia (Schwaegr.) Dix. Bastow (1887) as Hypnum cochlearifolium; Rodway (1914) as Weymouthia billardieri.

var. billardieri (Hampe) Dix. Sainsbury (1955f) did not consider this variety to be justified.

Weymouthia mollis (Hedw.) Broth. Bastow (1887) as Meteorium molle; Rodway (1914).

Weymouthia billardieri (Hampe) Broth. = Weymouthia cochlearifolia var. billardieri

\section{MITTENIACEAE}

Mittenia plumula (Mitt.) Lindb. Mitten (1882); Bastow (1887) as Mniopsis plumula; Rodway (1914).

Mniopsis plumula Mitt. $=$ Mittenia plumula

\section{MNIACEAE}

Mnium longirostre Brid. (Australian records) = Plagiomnium novaezealandiae. See Doubtful and Excluded Records.

Mnium rostratum Schrad. (Australian records)= Plagiomnium novaezealandiae. See Doubtful and Excluded Records.

Plagiomnium rostratum (Schrad.) T.Kop. (Australian records) = Plagiomnium novaezealandiae. See Doubtful and Excluded Records.

\section{NECKERACEAE}

Calyptothecium buftonii Broth. et Geh. = Rhabdodontium buftonii (Pterobryaceae)

Cladomnion, see Ptychomniaceae

Homalia pulchella Hook. f. et Wils. = Homalia punctata

Homalia punctata (Hook.f. et Wils.) Wijk et Marg. Jaeger \& Sauerbeck (1877); Brotherus (1906) as H. pulchella. There are no collections held in $\mathrm{HO}$ to support these early records.

Neckera hymenodonta C.Muell. $=$ Neckera pennata

Neckera pennata Hedw. Mitten 1882 as N. hymenodonta; Bastow (1887).

Neckera rivalis Mitt. $=$ Thamnobryum pumilum

Porotrichum fruticosum Mitt. = Camptochaete fruticosa . See Doubtful and Excluded Records.

Porotrichum vagum (Hornsch. ex C. Muell.) Mitt. = Camptochaete vaga (Lembophyllaceae)

Thamnium pandum (Hook. f. et Wils.) Jaeg. = Thamnobryum pandum.

Thamnium pumilum (Hook.f. et Wils.) Kindb. = Thamnobryum pumilum

Thamnium tenerascens Burchard = Thamnobryum pumilum

Thamnobryum pandum (Hook.f. et Wils.) Stone et Scott. Kindberg (1902) as Thamnium pandum. Scott \& Stone (1976) did not indicate that this occurs in Tasmania; however, a Tasmanian collection (D. McVean 26768) is held in CBG.

Thamnobryum pumilum (Hook.f. et Wils.) Nieuwl. Bastow 1887 as Isothecium pumilum; Rodway (1914) as Thamnium pumilum

\section{NEMATACEAE SE EPHEMEROPSIDACEAE}

\section{ORTHOTRICHACEAE}

Amphidium cyathicarpum (Mont.) Broth. Mitten (1860) as Didymodon cyathicarpus; Scott \& Stone (1976). 
Macrocoma tenue (Hook. et Grev.) Vitt. Bastow (1887) as Macromitrium microphyllum; Rodway (1914) as Macromitrium eucalyptorum; Vitt (1980b).

ssp. tenue

Macromitrium archeri Mitt. in Hook. Mitten (1860); Bastow (1887); Rodway (1914)

Macromitrium eucalyptorum C.Muell. et Hampe = Macrocoma tenue

Macromitrium ligulaefolium Broth. Vitt \& Ramsay (1985), an uncommon species in Tasmania.

Macromitrium longirostre (Hook.) Schwaegr. Bastow (1887); Rodway (1914); Weymouth \& Rodway (1922) as Macromitrium rodwayi.

Macromitrium microphyllum (Hook. et Grev.) Brid. = Macrocoma tenue ssp. tenue

Macromitrium microstomum (Hook. et Grev.) Schwaegr. Bastow (1887); Rodway (1914) as Macromitrium weymouthii.

Macromitrium pusillum Mitt. in Hook. = Macromitrium archeri

Macromitrium rodwayi Dix. in Weym. et Rodw. = Macromitrium longirostre

Macromitrium subulatum* Mitt. Mitten (1882) described from Bass' Straits (Tasmania?); Vitt \& Ramsay (1985). Known only from the type, collected by Milne, no specific habitat given.

Macromitrium tasmanicum Broth.' = Macromitrium microstomum

Macromitrium tenue (Hook. et Grev.) Brid. = Macrocoma tenue ssp. tenue

Macromitrium torquatulum (C.Muell.) C.Muell. et Broth. = Macromitrium longirostre

Macromitrium weymouthii Broth. = Macromitrium microstomum

Orthotrichum lateciliatum Vent. = Orthotrichum tasmanicum var. tasmanicum

Orthotrichum lawrencii Mitt. in Hook. = Orthotrichum tasmanicum var. tasmanicum

Orthotrichum rupestre Schleich. ex Schwaegr. Sainsbury (1955c).

Orthotrichum tasmanicum Hook.f. et Wils. Mitten (1882); Rodway (1914).

var. tasmanicum

Ulota anceps Vent. = Ulota viridis

Ulota appressa Mitt. = Ulota viridis

Ulota cochleata Vent. = Ulota viridis

Ulota dixonii Malta. Sainsbury (1955c). Rare; there are no collections in $\mathrm{HO}$.

Ulota laticiliata Malta. Sainsbury (1955c). Rare; there are no collections in $\mathrm{HO}$.

Ulota lutea (Hook.f. et Wils.) Mitt. Mitten (1860); Rodway (1914).

Ulota membranata Malta. Sainsbury (1955c).

Ulota viridis Vent. Weymouth (1894); Rodway (1914).

Ulota weymouthii Burchard = Ulota lutea

Zygodon anomalus Dozy et Molk. = Zygodon reinwardtii

Zygodon brownii Schwaegr. = Zygodon intermedius

Zygodon cyathicarpus Mont. = Amphidium cyathicarpum

Zygodon hookeri Hampe. Sainsbury (1955c).

Zygodon intermedius B.S.G. Mitten (1882); Rodway (1914).

Zygodon menziesii (Schwaegr.) Amott. Mitten (1882).
Zygodon minutus C.Muell, et Hampe. Bastow (1887); Sainsbury (1955c). Rare, no collections in HO.

Zygodon obtusifolius Hook. Weymouth (1903). Rare, western Tasmania, epiphytic.

Zygodon reinwardtii (Hornsch.) Braun. Mitten (1882). Rare; known only from southeastern Tasmania.

Zygodon rodwayi* Broth. in Rodw. Rodway (1914), but Sainsbury (1955c) made no reference to this in dealing with Rodway's herbarium.

\section{PHYLlogoniaCEAE}

Catagonium nitens (Brid.) Card. = Catagonium politum Catagonium politum (Hook.f. et Wils.) Dus. ex Broth. Rodway (1914); Sainsbury (1956b).

\section{Plagiotheciaceae}

Isopterygium see Hypnaceae

Plagiothecium denticulatum (Hedw.) B.S.G. Mitten (1882); Rodway (1914) as P. lamprostachys.

Plagiothecium lamprostachys (Hampe) Jaeg. = Plagiothecium denticulatum

\section{Pleurophascaceae}

Pleurophascum grandiglobum Lindb. Bastow (1887); Rodway (1914). 1nfrequent in buttongrass sedgeland and wet subalpine heathland. Restricted to the southern and western parts of Tasmania and New Zealand.

\section{POLYTRICHACEAE}

Atrichum androgynum (C.Muell.) Jaeg. Mitten (1882) as A. muelleri; Rodway (1914) as Catharinea muelleri.

Atrichum muelleri (C.Muell. et Hampe) Jaeg. = Atrichum androgynum

Atrichum pusillum (C.Muell.) Par.= Atrichum androgynum

Catharinea muelleri C.Muell. et Hampe = Atrichum androgynum

Pogonatum alpinum (Hedw.) Roehl. = Polytrichastrum alpinum

Pogonatum australasicum (C.Muell. et Hampe) Jaeg. = Pogonatum subulatum

Pogonatum gulliveri (Hampe) Jaeg. Mitten (1882); Hyvönen (1989) considered that this moss belongs in Rhizogoniaceae.

Pogonatum subulatum (Brid.) Brid. Scott \& Stone (1976).

Polytrichadelphus lagenaceus (C.Muell.) Par. = Polytrichadelphus magellanicus

Polytrichadelphus magellanicus (Hedw.) Mitt. Mitten (1860); Rodway (1914).

Polytrichastrum alpinum (Hedw.) G.L.Smith. Mitten (1882) as Pogonatum alpinum; Bastow (1887); Rodway (1914) as Polytrichum alpinum.

Polytrichum alpinum Hedw. = Polytrichastrum alpinum

Polytrichum australe Hook.f. et Wils. = Psilopilum australe

Polytrichum commune Hedw. Mitten (1882); Bastow (1887); Rodway (1914).

Polytrichum crispulum Hook.f. et Wils. = Psilopilum crispulum

Polytrichum croceum* Hampe. Watts \& Whitelegge (1902) but no collection or locality was cited. There are no collections held in $\mathrm{HO}$. 
Polytrichum formosum Hedw. Ratkowsky \& Ratkowsky (1983).

Polytrichum juniperinum Hedw. Mitten (1882); Bastow (1887) Rodway (1914).

*var, australe C.Muell.

Polytrichum lycopodioides* C.Muell. Watts \& Whitelegge (1902) but no locality was specified. There are no collections held in $\mathrm{HO}$.

Polytrichun magellanicum Hedw. = Polytrichadelphus magellanicus

Polytrichum tasmaniae C.Muell. = Polytrichum juniperinum

Psilopilum ausirale (Hook.f. et Wils.) Mitt. Mitten (1882); Rodway (1914).

Psilopilum crispulum (Hook.f. et Wils.) Mitt. Mitten (1882); Rodway (1914).

\section{POTTIACEAE}

Acaulon apiculatum Mitt. $=$ Acaulon integrifolium

Acaulon integrifolium C.Muell. Mitten (1882) as A. turgidum. Anomodon see Thuidiaceae

Astomum cylindricum (Tayl.) Mitt. = Tetrapterum cylindricum

Astomum weymouthii (C.Muel1.) Broth. = Tetrapterum weymouthii

Barbula australasiae (Hook. et Grev.) Brid. = Didymodon australasiae

Barbula calycina Schwaegr. Mitten (1882); Rodway (1914).

Barbula chrysopus* C.Muell, Scott \& Stone (1976).

Barbula crinita Schultz. Mitten (1882) as Tortula pseudopilifera.

Barbula hampeana Par. Scott \& Stone (1976).

Barbula hornschuchiana Schultz. Catcheside (1980).

Barbula knightii (Mitt.) Reichdt. = Tortella knightii

Barbula luteola (Mitt.) Par. Bastow (1887) as Tortula pungens.

Barbula pseudopilifera C. Muell. et Hampe = Barbula crinita

Barbula pungens Jaeg. $=$ Barbula luteola

Barbula rubella (Hook.f. et Wils.) Jaeg. = Tortula princeps

Barbula subtorquata C.Muell. et Hampe = Didymodon subtorquatus

Barbula torquata Tayl. = Didymodon torquatus

Barbula unguiculata Hedw. Weymouth (1903).

Bryoerythrophyllum binnsii (R.Br.ter.) Wijk et Marg. = Bryoerythrophyllum jamesonii

Bryoerythrophyllum jamesonii (Tayl.) Crum. Ratkowsky \& Ratkowsky (1983).

Calyptopogon mnioides (Schwaegr.) Broth. Bastow (1887) as Tortula mnioides; Rodway (1914) as Streptopogon crispatus.

Desmatodon convolutus (Brid.) Grout. Bastow (1887) as D. nervosus.

Desmatodon nervosus DeNot. $=$ Desmatodon convolutus

Desmatodon recurvatus (Hook.) Mitt. Mitten (1882) as Tortula recurvata.

Didymodon australasiae (Hook. et Grev.) Zand. Bastow (1887) as Tortula australasiae.

Didymodon cyathicarpus (Mont.) Mitt. = Amphidium cyathicarpum (Orthotrichaceae)

Didymodon papillatus Hook.f. et Wils. = Triquetrella papillata
Didymodon subtorquatus (C.Muell. et Hampe) Catches. Weymouth (1894) as Barbula subtorquata.

Didymodon tasmanicus (Hook.f.) Mitt. = Tridontium tasmanicum

Didymodon torquatus (Tayl.) Catches. Bastow (1887) as Tortula torquata.

Eucladium tasmanicum Broth. ex Rodw. = ? Gymnostomum calcareum

Gymnostomum aeruginosum Sm. Streimann \& Curnow (1989).

Gymnostomum calcareum Nees et Hornsch. Bastow (1887). Streimann \& Curnow (1989) considered synonymous with $G$. aeruginosum.

Leptodontium papillatum Hook.f. et Wils. ex Par. = Triquetrella papillata

Phascum apiculatum Hook.f. et Wils. = Acaulon integrifolium

Phascum cristatum Hook.f. et Wils. = Ephemerum cristatum (Ephemeraceae)

Phascum cylindricum Tayl. $=$ Tetrapterum cylindricum

Phascum gracilentum Mitt. = Pleuridium nervosum (Ditrichaceae)

Phascum tasmanicum Dix.et Rodw. Dixon \& Rodway (1923). var. tasmanicum. Stone (1989).

Phascum weymouthii C.Muell. $=$ Tetrapterum weymouthii

Pottia davalliana (Sm.) C.Jens. Scott \& Stone (1976).

Pottia drummondii (Wils.) Willis. Scott \& Stone (1976). var, obscura Willis. Catcheside (1980).

Pottia heimii (Hedw.) Hampe. Weymouth \& Rodway (1922); Sainsbury (1953b).

Pottia meibourniana Dix. ex Weym. et Rodw. = Pottia truncata

Pottia starckeana (Hedw.) C.Muell. Scott \& Stone (1976).

Pottia subphyscomitrioides Broth. = Pottia truncata

Pottia tasmanica* Broth. Scott \& Stone (1976).

Pottia truncata (Hedw.) B.S.G. Sainsbury (1953b).

Streptopogon crispatus (Hampe) Jaeg. = Calyptopogon mnioides

Tetrapterum cylindricum (Tayl.) Jaeg. Bastow (1887) as Phascum cylindricum.

Tetrapterum weymouthii* (C.Muell.) Broth. Watts \& Whitelegge (1902) as Astomum weymouthii.

Tortella calycina (Schwaegr.) Dix. = Barbula calycina

Tortella inclinata (Hedw.f.) Limpr. Mitten (1882) as Tortula nervosa.

Tortella knightii (Mitt.) Broth. Mitten (1882) as Tortula knightii; Rodway (1914).

Tortula anderssonii Aongstr. Ramsay et al. (1986) as T. bealeyensis.

Tortula antarctica (Hampe) Wils. = Tortula princeps

Tortula atrovirens $(\mathrm{Sm}$.$) Lindb. =$ Desmatodon convolutus

Tortula australasiae Hook. et Grev. = Didymodon australasiae

Tortula bealeyensis $\mathrm{R} . \mathrm{Br} .=$ Tortula anderssonii Lightowlers (1986).

Tortula brachytricha (C.Muel1.) Broth. = Tortula princeps

Tortula calycina (Schwaegr.) Hook. et Grev. = Barbula calycina

Tortula cuspidata Hook.f. et Wils. = Tortula princeps

Tortula elaphrotricha (C.Muell.) Broth. $\mathrm{ex}$ Watts et Whitel. = Tortula muralis 
Tortula knightii Mitt. = Tortella knightii

Tortula latrobeana (C.Muell.) Mitt. Mitten (I882); Bastow (1887), but there are no collections in $\mathrm{HO}$ to support these early records.

Tortula luteola Mitt. = Barbula luteola

Tortula mnioides (Schwaegr.) Mont. = Calyptopogon mnioides

Tortula muelleri Hook.f. et Wils. = Tortula princeps

Tortula muralis Hedw. Weymouth (1894); Rodway (1914).

Tortula pagorum (Milde) DeNot. Ratkowsky \& Ratkowsky (1983). Uncommon, often growing with Tortula papillosa in drier habitats.

Tortula panduraefolia (C.Muell. et Hampe) Broth. Weymouth (1903); Rodway (1914) places in synonomy with $T$. princeps.

Tortula papillosa Wils. Bastow (1887); Rodway (1914).

Tortula princeps DeNot. Bastow (1887) as T. muelleri; Rodway (1914).

Tortula pseudopilifera (C.Muell. et Hampe) Mitt. = Barbula crinita

Tortula pungens Hook.f. et Wils. = Barbula luteola

Tortula recurvata Hook. = Desmatodon recurvatus

Tortula rubella Hook.f. et Wils. = Tortula princeps

Tortula rubra Mitt. in Hook.f. Ratkowsky \& Ratkowsky (1983).

Tortula subtorquata (C.Muell. et Hampe) Mitt. = Didymodon subtorquatus

Tortula torquata (Tayl.) Wils. = Didymodon torquatus

Trichostomopsis australasiae (Hook. et Grev.) H.Robinson = Didymodon australasiae

Trichostomum australe Mitt. = Ditrichum strictum (Ditrichaceae)

Trichostomum cylindricarpum (C.Muell.) Wils. = Ditrichum cylindricarpum (Ditrichaceae)

Trichostomum elongatum Hook,f. et Wils. = Ditrichum cylindricarpum (Ditrichaceae)

Trichostomum laxifolium Hook.f. et Wils. = Ditrichum difficile (Ditrichaceae)

Trichostomum oldfieldii Mitt. = Ditrichum difficile (Ditrichaceae)

Trichostomuni setosum Hook.f. et Wils. = Ditrichum difficile (Ditrichaceae)

Tridontium tasmanicum Hook.f. Sainsbury (1953b).

Triquetrella papillata (Hook.f.et Wils.) Broth. Bastow (1887) as Didymodon papillatus; Rodway (1914) as Leptodontium papillatum.

Weissia calcarea (Nees et Hornsch.) C.Mucll. = Gymnostomum calcareum

Weissia controversa Hedw. Mitten (1882); Rodway (1914) as Weissia flavipes.

Weissia flavipes Hook.f. et Wils. = Weissia controversa

Weissia microcarpa Hook.f. et Wils. = Dicranoweisia microcarpa (Dicranaceae)

Weissia weymouthii C.Muell. = Weissia controversa. Sainsbury (1953b).

\section{PTEROBRYACEAE}

Calyptothecium buftonii Broth. et Geh. = Rhabdodontium buftonii

Pterobryum planifolium (Hedw.) Mitt. = Trachyloma planifolium
Pulchrinodusinflatus (Hook.f.et Wils.) Allen. Allen (1987b). A new record. Rare in southwestern Tasmania.

Rhabdodontium buftonii* (Broth. et Geh.) Broth. Weymouth (1896) as Calyptothecium buftonii; Rodway (1914). This moss was originally known from two collections of the late 19 th century and not found again until 1976, Norris \& Montalvo (1981). Rare, known from rocks in rapid streams in western Tasmania.

Trachyloma diversinerve Hampe. Miller \& Manuel (1982).

Trachyloma planifolium (Hedw.) Brid. Mitten (1882) as Pterobryum planifolium; Rodway (1914).

\section{PTYCHOMITRIACEAE}

Glyphomitrium latifolium Broth. $=$ Tridontium tasmanicum (Pottiaceae)

Ptychomitrium acutifolium Hook.f. et Wils. = Ptychomitrium australe

Ptychomitrium australe (Hampe) Jaeg. Bastow (1887) as P. acutifolium.

Ptychomitrium mittenii Jaeg. Bastow (1887) as $P$. serratum. Ptychomitrium serratum (Mitt.) Hook.f. et Wils. = Ptychomitrium mittenii

\section{PTYCHOMNIACEAE}

Cladomnion sciuroides (Hook.) Wils. = Glyphothecium sciuroides

Caldomion setosum $($ Hedw.) Wils. $=$ Cyrtopus setosus. See Doubtful and Excluded Records.

Cladomnion tasmanicum C.Muell. = Glyphothecium sp.

Glyphothecium sciuroides (Hook.) Hampe. Bastow (1887) as Cladonnion sciuroides; Rodway (1914).

Hampeellaalaris (Dix. et Sainsb.) Sainsb. Sainsbury (1955f). Ptychomnium aciculare (Brid.) Mitt. Mitten (1882); Bastow (1887) as Hypnum aciculare; Rodway (1914).

\section{RACOPILACEAE}

Racopilum australe Hook.f. et Wils. = Racopilum convolutaceum

Racopilum convolutaceum(C.Muell.) Reichdt. Mitten(1882) as $R$. strumiferum; Bastow (1887) as $R$. australe; Rodway (1914) as R. cristatum.

Racopilum cristatum Hook.f. et Wils. = Racopilum convolutaceum

Racopilum strumiferum C.Muell. = Racopilum convolutaceum. Scott \& Stone (1976)

Racopilum tomentosum (Hedw.) Brid. Jaeger \& Sauerbeck (1876). Scott \& Stone (1976) suggested that this species may be the same as $\boldsymbol{R}$. convolutaceum.

\section{RHIZOGONIACEAE}

Goniobryum subbasilare (Hook.) Lindb. Bastow (1887) as Rhizogonium subbasilare; Rodway (1914).

Hymenodon pilifer Hook.f. et Wils. Bastow (1887); Rodway (1914).

Pyrrhobryum bifarium (Hook.) Manuel. Mitten (1882); Bastow (1887); Rodway (1914) as Rhizogonium bifarium.

Pyrrhobryum mnioides (Hook.) Manuel. Bastow (1887); Rodway (1914) as Rhizogonium mnioides.

Pyrrhobryum parramattense (C.Muell.) Manuel. Scott \& Stone (1976) as Rhizogonium parramattense. 
Pyrrhobryum spiniforme (Hedw.) Mitt. Bastow (1887); Rodway (1914); Sainsbury (1955d) as Rhizogonium spiniforme.

Rhizogonium alpestre* C.Muell. Watts and Whitelegge (1906). According to Scott \& Stone (1976) the status of this taxon is in question.

Rhizogonium aristatum Hampe = Rhizogonium pennatum var. aristatum

Rhizogonium bifarium (Hook.) Schimp. = Pyrrhobryum bifarium. Manuel (1980).

Rhizogonium distichum (Sw.) Brid. Mitten (1882); Bastow (1887); Rodway (1914).

Rhizogonium hookeri (C.Muell.) Mitt. = Pyrrhobryum mnioides

Rhizogonium mnioides (Hook.) Wils, in Hook. = Pyrrhobryum mnioides. Manuel (1980).

Rhizogonium mossmanianum (C.Muell.) Jaeg. = Pyrrhobryum mnioides

Rhizogonium novaehollandiae (Brid.) Brid. Bastow (1887); Rodway (1914).

Rhizogonium parramattense (C.Muell.) Reichdt.= Pyrrhobryum parramattense. Manuel (1980).

Rhizogonium pellucidum (Mitt.) Jaeg. = Goniobryum subbasilare

Rhizogonium pennatum Hook f. et Wils.

var. aristatum (Hampe) Dix. Mitten (1882); Rodway (1914) treated at specific rank; Sainsbury (1955d).

Rhizogonium spiniforme (Hedw.) Bruch = Pyrrhobryum spiniforme

Rhizogonium subbasilare (Hook.) Schimp. = Goniobryum subbasilare

\section{RHYTIDIACEAE}

Rhytidiadelphus squarrosus (Hedw.) Warnst. A new record for Tasmania and apparently the Southern Hemisphere. One collection from west coast of Tasmania (A.J. Fife 8969).

\section{SELIGERIACEAE}

Blindia arcuata Mitt. = Blindia magellanica

Blindia ferruginea (Mitt. ex Wils.) Broth. = ?Dicranoweisia sp. (Dicranaceae). Identity uncertain, Bartlett \& Vitt (1986).

Blindia magellanica Schimp. in C.Muell. Mitten (1882) as B. arcuata; Sainsbury (1955b).

Blindia robusta Hampe. Weymouth (1894).

Blindia tasmanica Sainsb. = Blindia robusta

Blindia tenuifolia Mit. = Blindia robusta

\section{SEMATOPHYLLACEAE}

Acanthocladium extenuatum (Brid.) Mitt. = Wijkia extenuata Rhaphidorrhynchium amoenum (Hedw.) Fleisch. Bastow (1887) as Hypnum amoenum; Sainsbury (1956b) as Sematophyllum antoenum.

Rhaphidorrhynchium callidioides (C.Muell.) Broth. Weymouth (1894) as Rhaphidostegiam callidioides.

Rhaphidorrhynchium calliferum (Geh. et Hampe) Fleisch. Rodway (1914) as Rhaphidostegium calliferum; Sainsbury (1956b) considered synonomous with R. amoenum
Rhaphidorrhynchium jolliffii (Hook,f.) Broth. Mitten (1860) as Stereodon jolliffii; Bastow (1887) as Hypnum jolliffii.

Rhaphidorrhynchum cerviculatum (Hook.f. et Wils.) Mitt. = Sematophyllum leucocytus

Rhaphidorrhynchum homomtallum (Hampe) Mitt. = Sematophyllum homomallum

Rhaphidorhynchum tenuirostre $($ Hook.) Mitt. = Sematophyllum uncinatum

Rhaphidostegium callidioides (C.Muell.) Jaeg. = Rhaphidorrhynchium callidioides

Rhaphidostegium calliferum Geh. et Hampe = Rhaphidorrhynchium calliferum

Rhaphidostegium contiguum (Mitt.) Par. = Sematophyllum contiguum

Rhaphidostegium crassiusculum (Brid.) Jaeg. = Sematophyllum crassiusculum

Rhaphidostegium cyparioides (Brid.) Jaeg. = Rhaphidorrhynchium amoenum

Rhaphidostegium jolliffii (Hook.f.) Jaeg. = Rhaphido. rrhynchium jolliffii

Sematophyllum amoenum (Hedw.) Mitt. = Rhaphidorrhynchium amoenum

Sematophyllum caespitosum (Hedw.) Mitt. Dixon (1950) based on a collection made in 1824 .

Sematophyllum crassiusculum (Brid.) Broth. Rodway (1914) as Rhaphidostegium crassiusculum

Sematophyllum contiguum (Mitt.) Mitt. in Seeman. Bastow (1887) as Hypnum contiguum; Sainsbury (1956b).

Sematophyllum homomallum (Hampe) Broth. Mitten (1882) as Rhaphidorrhynchum homomallum; Sainsbury (1956b).

Sematophyllum jolliffii (Hook.f.) Dix. = Rhaphidorrhynchium jolliffii

Sematophyllum leucocytus (C.Muell.) Sainsb. Mitten (1882) as Rhaphidorrhynchum cerviculatum; Bastow (1887) as Hypnum cerviculatum; Sainsbury (1956b).

Sematophyllum subcylindricum (Broth. ex Fleisch.) Sainsb. Scott \& Stone (1976).

Sematophyllum tenuirostre (Hook.) Dix. = Sematophyllum uncinatum

Sematophyllum uncinatum Stone et Scott. Mitten (1882) as Rhaphidorrhynchum tenuirostre; Scott \& Stone (1976).

Wijkia extenuata (Brid.) Crum. Mitten (1882) as Acanthocladium extenuatum.

\section{SPHAGNACEAE}

The taxonomy and nomenclature of Australian Sphagnum species is confused and awaits a thorough revision.

Sphagnum antarcticum Mitt. = Sphagnum australe

Sphagnum australe Mitt. Mitten (1882).

Sphagnum beccarii Hampe. Streimann \& Curnow (1989) list for Tasmania. Watts (1912) made no reference to the species, while Willis (1952) records for Victoria only. Sphagnum brotherusil Warnst. = Sphagnum falcatulum Sphagnum centrale C.Jens. Weymouth (1903).

Sphagnum confertum Mitt. = Sphagnum australe

Sphagnum compactum Lamk. et Cand. Mitten (1882).

Sphagnum contortum Schult $=$ Sphagnum cymbifolioides Sphagnum cristatum Hampe. Scott \& Stone (1976).

Sphagnum cuspidatum Ehrh. ex Hoffm. Weymouth (1903). Sphagnum cymbifolioides C.Muell. Mitten (1882). 
Sphagnum cymbifolium (Ehrh.) Hedw. = Sphagnum palustre Sphagnum drepanocladum Warnst. $=$ Sphagnum falcatulum Sphagnum falcatulum Besch. Scott \& Stone (1976).

Sphagnum leucobryoides Yamaguchi, Seppelt et Iwatsuki. Yamaguchi et al. (1990). A newly described species, confined to southwestern Tasmania.

Sphagnum macrocephalum Warnst. = Sphagnum australe Sphagnum maximum Warnst. = Sphagnum cristatum Sphagnum molliculum Mitt. = Sphagnum cymbifolioides Sphagnum moorei Warnst. = Sphagnum cymbifolioides Sphagnum mossmanianum C.Muell. = Sphagnum cymbifolioides

Sphagnum novaezealandiae Mitt. = Sphagnum cymbifolioides

Sphagnum orthociadum Burch = Sphagnum australe

Sphagnum palustre L. Mitten (1882) as S. cymbifolium.

Sphagnum pseudorufescens Warnst. = Sphagnum cymbifolioides

Sphagnum rodwayi Warnst. = Sphagnum falcatulum

Sphagnum serrulatum Warnst. = Sphagnum falcatulum

Sphagnum subbicolor Hampe (Australian records) = Sphagnum cristatum

Sphagnum submolliculum Warnst. = Sphagnum cymbifolioides

Sphagnum subsecundum Nees (Australian records) = Sphagnum cymbifolioides

Sphagnum trichophyllum Warnst. = Sphagnum falcatulum

Sphagnum weymouthii Warnst. ex Rodw. = Sphagnum australe

\section{SPLACHNACEAE}

Dissodon plagiopus (Mont.) C.Muell. = Tayloria octoblepharum

Splachnum callophyllum (C.Muell.) Wils. = Tayloria callophylla. See Doubtful and Excluded Records.

Splachnum gunnii Wils. = Tayloria gunnii

Splachnum octoblepharum Hook. = Tayloria octoblepharum

Tayloria gunnii* (Wils.) Willis. Mitten (1882) as Splachnum gunnii; Willis (1950). An uncommon rainforest moss.

Tayloria obtussisima Broth. $=$ ?Tayloria gunnii

Tayloria octoblepharum (Hook.) Mitt. Mitten (1882); Rodway (1914).

var. major (Hook.f. et Wils.) Watts et Whitel.

var. pyriformis (Hook.f. et Wils.) Watts et Whitel.

Tayloria tasmanica* (Hampe) Broth. Weymouth (1894) as Tetraplodon tasmanicus; Willis (1950). Terrestrial, cushion forming, in buttongrass sedgeland of southwestern Tasmania.

Tetraplodon tasmanicus Hampe = Tayloria tasmanica

\section{THUIDIACEAE}

Anomodon tasmanicus Broth. Weymouth (1903).

Thuidiopsis furfurosa (Hook.f. et Wils.) Fleisch. = Thuidium furfurosum

Thuidium furfurosum (Hook.f. et Wils.) Reichdt. Mitten (1882).

var. sparsum (Hook.f. et Wils.) Sainsb. = Thuidium sparsum var. sparsum

Thuidium laeviusculum (Mitt.) Jaeg. Rodway (1914); Sainsbury (1956a).

Thuidium sparsum (Hook.f. et Wils.) Reichdt. var. hastatum (Mitt.) Touw et Falter. Rodway (1914) as T. stuartii; Touw \& Falter (1989). Restricted in Tasmania to the southeast but occurs also in southern Australia.

var. sparsum Weymouth (1894); Touw and Falter (1989).

Thuidium stuartit* (C.Muell.) Jaeg. = Thuidium sparsum var. hastatum

Thuidium unguiculatum (Hook.f. et Wils.) Fleisch. = Thuidium sparsum var. sparsam

\section{Doubtful and Excluded Records}

Acaulon crassinervium C.Muell. = Phascum robustum var, crassinervium. Recorded for Tasmania by Scott \& Stone (1976) but excluded by Stone (1989).

Andreaea australis Mitt. Schultze-Motel (1970) doubts this record for Tasmania.

Brachymenium coarctatum Bosch et Lac. Watts \& Whitelegge (1906), but the locality given is probably not in Tasmania.

Bryum australe Hampe. No collections in HO; Australian occurrence uncertain, see Scott \& Stone (1976).

Bryum rubiginosum Hook.f. et Wils. Rodway (1914). See Sainsbury (1955d).

Camptochaete angustata (Mitt.) Reichdt. Bastow (1887) as Isothecium angustatum. However, Sainsbury (1955f) made no mention of this species occuring in Tasmania and considered it as endemic in New Zealand.

Camptochaete fruticosa Par. Mitten (1882) as Porotrichum fruticosum; Bastow (1887) as Isothecium fruticosum. However, Sainsbury (1955f) made no mention of this occuring in Tasmania.

Catharomnion ciliatum (Hedw.) Wils. Mitten (1882) as Hypopterygium ciliatum; Bastow (1887). Rodway (1914) suggested it was recorded in error as there were no collections available. Sainsbury (1956a) and Scott \& Stone (1976) also suggest recorded in error.

Ceratodon crassinervis Lorentz. Occurrence in Tasmania considered tentative (Scott \& Stone 1976). There are no collections in HO.

Ceratodon stenocarpus B.S.G. Mitten (1882); Rodway (1914); Scott \& Stone (1976) stated the occurrence should be considered tentative. There are no collections in HO.

Cladomnion ericoides (Hook.) Wils. Although Streimann \& Curnow (1989) list for Tasmania, Sainsbury (1955a) gave this as a New Zealand species only.

Climacium dendroides (Hedw.) Web. et Mohr. Martin (1946) lists for Tasmania; however, Stone (1985) recorded new for Australia only in Victoria.

Cyptodon dilatatus (Hook.f. et Wils.) Par. et Schimp. Listed for Tasmania by some authors as a result of Rodway's misinterpretation of C. parvula. Scott \& Stone (1976) do not record for Tasmania.

Cyrtopus setosus (Hedw.) Hook.f. Bastow (1887) as Cladomnion setosum. Rodway (1914) indicated there are no Tasmanian collections and possibly reported in error

Dicranoweisia cirrata (Hedw.) Lindb. See Holomitrium cinratum.

Ditrichum brachycarpum Hampe. Streimann \& Curnow (1989) list for Tasmania; howcver, Seppelt (1982) did not record for Tasmania. 
Entosthodon cuspidatus Kiaer in C. Muell. non. nud. Weymouth (1896)

Eriopus tasmanicus Broth. Weymouth (1903); Rodway (1914) but Sainsbury (1956a) considered these collections to be referable to E. apiculatus (=Calyptrochaeta apiculata).

Fissidens crassipes Wils. ex B.S.G. Streimann \& Curnow (1989) list for Tasmania; however, Willis (1955a) reported this moss from southeastern mainland Australia only.

Funaria radians (Hedw.) C.Muell. Two old collections only held in $\mathrm{HO}$ which are named without authority.

Grimmia funalis (Schwaegr.) B.S.G. Mitten (1882); Bastow (1887), but no collections at HO. Scott \& Stone suspect this is a misnomer for G. trichophylla.

Grimmia ovalis (Hedw.) Lindb. Record based on Sainsbury ( 1955 c) but Scott \& Stone (1976) consider its occurence in Tasmania doubtful.

Grimmia readeri Broth. Scott \& Stone (1976) list only for Victoria. Streimann \& Curnow (1989) list for Tasmania, possibly in error.

Holomitrium cirratum (Hedw.) Mitt. Recorded by Mitten (1860) but Scott \& Stone (1976 - as Dicranoweisia cirrata (Hedw.) Lindb.) consider this to be in error.

Homalia falcifolia Hook.f. et Wils. Mitten (1882); Rodway (1914). Sainsbury (1955f) considered this record doubtful. There are no collections in HO to support the record.

Hypnodendron colensoi(Hook. f. et Wils.) Mitt. Mitten (1882); Bastow (1887) as Isothecium colensoi. Touw (1971) considered these early records to be doubtful.

Hypopierygium conmuiatum C.Muell. Sainsbury (1955a); however, Scott \& Stone (1976) could find no record of this as a Tasmanian moss. There are no collections held in $\mathrm{HO}$.

Hypopterygium muelleri Hampe. Scott \& Stone (1976) record for NSW, QId and Lord Howe Island only. There are no collections held in $\mathrm{HO}$.

Leptostomum macrocarpum (Hedw.) Pyl. Bastow (1887). Sainsbury (1955d) and Scott \& Stone (1976) considered this record may be in error. No collections in $\mathrm{HO}$.

Mesotus acutus Mitt. Recorded for Australia by Mitten (1882) but not for Tasmania. Allen (1987a) doubts that this moss occurs in Tasmania.

Plagiomnium nov'aezealandiae (Col.) T.Kop. Weymouth \& Rodway (1922) as Mnium rostratum, but Sainsbury (1955d) considered to be misidentified. Koponen (1983) excludes the record for Tasmania.

Polytrichum novaehollandiae Jaeg. Streimann \& Curnow (1989) report for Tasmania but the record of Watts \& Whiteleggc (1902) gives the locality as Mt Wellington in Victoria.

Polvtrichum piliferum Hedw. Watts \& Whitelegge (1902) considered the record doubtful.

Racomirrium hypnoides Lindb. Rodway (1914) nom. illeg. = Racomitrium lanuginosum ssp. lanuginosum.

Tayloria callophylla (C.Muell.) Mitt. Mitten (1882). Rodway (1914) listed for Tasmania but indicated that the only collection was from New Zealand. Sainsbury (1955e) stated that it had been seen only from the North Island of New Zealand and that its occurrence in Tasmania was uncertain. There are no Tasmanian collections in $\mathrm{HO}$.
Thuidiumincompletopinnatum C.Muell. nom. nud. Weymouth (1894).

Weissia bicolor Hampe nom. nud. The plant so-named by Rodway is Dicranoweisiamicrocarpa, Sainsbury (1953).

Ulotafulva Brid. A misidentification; the Rodway material at HO has been redetermined as $U$. membranata Malta by Sainsbury.

\section{ACKNOWLEDGEMENTS}

We thank participants in the Australasian Bryological Workshop for their comments, corrections and additions to the initial checklist. Dr George Scott, Queen's College, University of Melbourne, offered many constructive comments and suggestions in the preparation of the manuscript. We are grateful to the Curator of the Tasmanian Herbarium for access to the collections in HO.

\section{REFERENCES}

AlLev, B.H., 1987a: A revision of the genus Mesotus (Musci: Dicranaceae). J. Bryol. $14: 441-452$.

ALlen, B.H., 1987b: A systematic account of Pulchrinodus inflatus (Musci: Pterobryaceae), genus novum. NZ J. Bot. 25: 335-342.

BARTLETT, J.K. \& VITT, D.H., 1986: A survey of species in the genus Blindia (Bryopsida, Seligeraceae). NZ J. Bot. 24: 203-246.

Bastow, R.A., 1887: Tasmanian mosses. Pap. Proc. R. Soc Tasm. (1886): 38-102.

BeEver, J.E., 1988: A key to the genera of New Zealand mosses. Nat. Mus. NZ Miscellaneous Series No. 18

Brotherus, V.F., 1901-09: Musci. In Engler, H.G.A. \& Prantl, K.A.E. (Eds): Die Naturlichen Pflanzenfamilien I(3): 277-1246. W. Engelmann, Leipzig.

Buchanan, A.M., MicGeary-Brown, A. \& Orchard, A.E., 1989: A census of the vascular plants of Tasmania. Tasmanian Herbarium Occ. Publ. 2.

Buck, W.R., I98I. A review of Cheilothela (Ditrichaeceae). Brittonia 33(3): $453-456$.

Catchesioe, D.G., 1980: MOSSES OF SOUTH AUSTRALHA Goverment Printer, Adelaide.

Catcheside, D.G. \& Frahm, J.-P., 1985: Additions to the Campylopus flora of Australia. J. Bryol. 13:359-367.

Cherchil.t, S.P. \& Buck, W.R., 1982: Taxonomic investigation of Leptotheca (Rhizogoniaceae). Brittonia 34: 1-11.

Crosby, M.R. \& Magill, R.E., 1981: A DICTIONARY OF MOSSES. Missouri Botanical Garden. 43pp

Dixon, H.N., 1950. Notes on the moss collections of the Royal Botanic Garden, Edinburgh. Part II. Notes R. Bot. Gard. Edinburgh (1948) 20: 93-102.

Dixon, H.N. \& Ronway, L., 1923: On Phascum tasmanicum Pap. Proc. R. Soc. Tasm. (1922): 25-26. 
DLNCAN, D. \& DALTON, P.J., 1982: Recolonization by bryophytes following fire. J. Bryol. 12: 53-63.

Fufischer, M., 1922. Kritische revision der Carl Mullerschen. Laubmoosgattungen IV. Hedwigia 63: 209-216.

Fratim, J.-P., 1987: A survey of the Campylopus species of Australia. J. Bryol. 14: 701-727.

HAMPE, E., 1872. Musci novi Australiae ex herbaria, a Doctore F. von Muller dissi. Linnaea 37: 513-519.

HaRRIS, S. \& RatKowsky, D.A., 1982: A new moss record for Tasmania lschyrodon lepturus (Tayl.). Schelpe. Tasm. Nat. 71: 7 .

Hyvönen, J., 1989: A synopsis of genus Pogonatum (Polytrichaceae, Musci). Acta Bot. Fenn. 138: 1-87.

JAEGER, A. \& SAUERBECK, F., 1876-79. GENERA ET SPECIES MUSCORUM SYSTEMATICE DISPOSITA SEU ADUMBRATIO FLORAE MUSCORUM TOTIUS ORBIS TERRARUM. VOLS 1, 2. Zollikofer, St Gallen (Switz.).( Reprinted 1973 from BER. THATIGK. ST GALLISCHEM NATURWISS. GES., 1870-75.)

Jarman, S.J., Kantvilas, G. \& Brown, M.J., 1988: Buttongrass moorland in Tasmania. Res. Rep. 2. Tasmanian Forest Research Council Inc., Hobart.

KANDA, H., 1977: A revision of the family Amblystegiaceae of Japan II. J. Sci. Hiroshima Univ. ser. B, div. 2 (Bot.) 16: 47-119.

Kantvilas, G, 1989: A checklist of Tasmanian lichens. Pap. Proc. R. Soc. Tasm. 123: 67-85.

Kindberg, N.C., 1902. Grundzuge einer Monographie der Laubmoos - Gattung, Thamnium. Hedwigia 41: 203-268.

Koponen, T., 1983: A synopsis of Mniaceae (Bryophyta) VIII. Taxa in Australia and New Zealand. Ann. Bot. Fenn. 20: 101-104.

LAWTON, E., 1973: Rhacomitrium crispulum and some related species, Bull. Torrey Bot. Club 100: 230-235.

Lightowlers, P.J., 1986: Taxonomy and distribution of the subantarctic species of Tortula. J. Bryol. 14: 281295.

ManUel., M.G., 1980. Miscellanea bryologia II. Classification of Rhizogonium Brid., Penzigiella hookeri Gangulee, and some nomina nuda. Cryptog. Bryol Lichenol. 1: 6772

MARTIN, W., 1946. Geographic range and internal distribution of the mosses indigenous to New Zcaland. Trans Proc. R. Soc. NZ 76: 162-184.

MeiJer, W., 1952: The genus Orthodontium. Acta Bot. Neerl. 1: 3-80.

Miller, H.A., Whittier, H.O. \& Whittier, B.A., 1978. Prodromus florae muscorum Polynesiae with a key to genera. Bryophytorum Biblioth. 16. J. Cramer, Vaduz.

Miller, N.G. \& Manuel, M.G., 1982. Trachyloma (Bryophytina, Pterobryaceae): A taxonomic monograph. J. Hattori Bot Lab. 51: 273-321.

Mitten, W., 1860: Description of some new species of musci from New Zealand and other parts of the southern hemisphere, together with an enumeration of the species collected in Tasmania by William Archer Esq.; arranged upon the plan proposed in the 'Musci Indiae Orientalis'. J. Linn. Soc., Bot. 4: 64 100 .
Mitren, W., 1882: Australian mosses. Trans. Proc. R. Soc. Vict. 19: 49-96.

Noguchi, A., 1985. The Isobryalian mosses collected by Dr Z. Iwatsuki in New Caledonia. J. Hattori Bor. Lab. 58 87-109.

Norris, D.H. \& Montalvo, A.M., 1981: The rediscovery of Rhabdodontium buftonii (Broth. \& Geh. in Broth.) Broth. Bryologist 84: 85-88.

OChyra, R., 1982. Kindbergia (Brachytheciaceae, Musci), a new name for Stokesiella (Kindb.) Robins., hom. illeg. Lindhergia 8: 53-54.

PARIS, E.G., 1894 98. Index bryologicus sive ENU MERATIO MUSCORUM HUCUSQUE COGNITARUM ADIUNCTUS SYNONYMIA DISTRIBUTIONEQUE GEOGRAPHICA LOCUPLETISSIMIS. P Klincksieck, Paris.

Ramsay, H.P., Streimann, H., Ratkowsky, A.V., Seppit, R., \& FIFE, A., 1986: Australian alpine bryophytes. In Barlow, B.A. (Ed.): FLORA AND FAUNA OF ALPINE AUSTRALASIA: AGES AND ORIGINS. CSIRO, Melboume: 301-335

Ratkowsky, D.A., 1980: New moss collections from Tasmania. Aust. Bryol. Newsl. 2: 2.

Ratkowsky, D.A., 1987: Checklist of the Tasmanian liverworts. Pap. Proc. R. Soc. Tasm. 121: 153-158.

Ratkowsky, D.A. \& Ratkowsky, A.V., 1982: The bryophytes of the Mt Wellington Range, Tasmania. Pap. Proc: R. Soc: Tasm. 116: 97-115.

RatKowsky, D.A. \& Ratkowsky, A.V., 1983: Some new additions to the bryoflora of Tasmania. Tasm. Nat. 73: $5-6$.

REImERs, H., 1926. Beiträge zur Bryophyten flora siidamerikas. I \& II. Hedwigia 66: 27-78.

Robinson, H., 1962. Generic revisions of North American Brachytheciaceae. Bryologist 65: 73-146.

RoDway, L., 1913-14: Tasmanian Bryophyta I. Mosses. Pap. Proc. R. Soc. Tasm. (1912): 3-24, 87-138; (1913): 177-263.

RODWAY, L., 1914: TASMANIAN BRYOPHYTA. VOL. I MOSSES. Royal Society of Tasmania, Hobart: $163 \mathrm{pp}$.

RoDway, L., 1915: Additions to the Tasmanian flora. Pap. Proc. R. Soc Tasm.: 104-107.

Rodway, L., 1916: Additions to the Bryophyte flora. Pap. Proc. R. Soc. Tasm.: 44-47.

SaINBbur, G.O.K., 1953a: Two new species of Tasmanian mosses. Vict. Nat. 70: 30-31.

Sainsbury, G.O.K., 1953b: Notes on Tasmanian mosses from Rodway's herbarium. Pap. Proc. R. Soc. Tasm. 87: $83-91$.

Sainsbury, G.O.K., 1955a: A handbook of the New Zealand mosses. Bull. R. Soc. NZ 5.

Sainsbliry, G.O.K., 1955b: Notes on Tasmanian mosses from Rodway's herbarium. II. Pap. Proc. R. Soc. Tasm. 89: $1-11$.

Sainsbury, G.O.K., 1955c: Notes on Tasmanian mosses from Rodway's herbarium. III. Pap. Proc. R. Soc. Tasm. 89: $13-20$.

SAINSBCRY, G.O.K., 1955d: Notes on Tasmanian mosses from Rodway's herbarium. IV. Pap. Proc. R. Soc. Tasm. 89: $21-35$. 
Sainsbury, G.O.K., 1955e: Notes on Tasmanian mosses from Rodway's herbarium. V. Pap. Proc. R. Soc. Tasm. 89: $37-43$.

SaINsBury, G.O.K., 1955f: Notcs on Tasmanian mosses from Rodway's herbarium. VI. Pap. Proc: R. Soc Tasm. 89: $45-53$

Sainsbury, G.O.K., 1956a: Notes on Tasmanian mosses from Rodway's herbarium. VII. Pap. Proc. R. Soc. Tasm. 90: $35-39$.

SaINsbury, G.O.K., 1956b: Notes on Tasmanian mosses from Rodway's herbarium. VIII. Pap. Proc. R. Soc. Tasm. 90: 41-47.

SCOTT, G.A.M. \& STONE 1.G., 1976: THE MOSSES OF SOUTHERN AUSTRALIA. Academic Press, London.

ScotT, G.A.M. \& STone, 1.G., 1979: In defence of Dawsonia superba Grev. Lindbergia 5: 71-72.

Schultze-Motel, W., 1970: Monographic de Laubmoosgattung Andreaea 1. Die costaten Arten. Willdenowia 6: 25-110

SEPPELT, R.D., 1981: Studies on the bryoflora of Macquarie 1sland. III. Collections, new moss additions and corrections. Bryologist 84(2): $249-252$.

Seppetr, R.D., 1982: A monographic revision of the genus Ditrichum (Musci: Ditrichaceae). 1. Australian and New Zealand species. J. Hattori Bot. Lab. 51: 99 150.

STONE, I.G., 1985. New records of mosses in Australia. J. Bryol. 13: $475-478$,

StOne, I.G., 1989: Revision of Phascum and Acaulon in Australia. J. Bryol. 15: 747-777

Stremann, H. \& CuRNow, J., 1989: CATALOGUE OF MOSSES OF AUSTRALIA AND ITS EXTERNAL TERRITORIES. Australian Flora and Fauna Series Number 10. Aust. Govt Publ. Serv., Canberra: $479 \mathrm{pp}$.

Touw, A., 1971: A taxonomic revision of the Hypnodendraceae (Musci). Blumea 19: 211-354.

Tolw, A. \& Falter-van den HaAK, L., 1989: A revision of the Australasian Thuidiaceae (Musci), with notes on species from adjacent regions. J. Hattori Bot. Lab. 67: $1-57$.

Vist, D.H., 1979. The Moss flora of the Auckland Islands, New Zealand, with a consideration of habitats, origins and adaptations. Can. J. Bot. 57: 2226-2263.

VirT, D.H., 1980a: A comparative study of Andreaea acutifolia, A. mutabilis, and A. rupestris. NZ J Bot. 18: $367-377$.

VITT, D.H., 1980b: The genus Macrocoma I. Typification of names and taxonomy of the species. Bryologist 83: $405-436$.

VITT, D.H. \& RAMSAY, H.P., 1985: The Macromitrium complex in Australasia (Orthotrichaceae: Bryopsida). Part I. Taxonomy and phylogenetic relationships. $J$. Hattori Bot. Lab. 59: 325-451.

WatTs, W.W., 1912. The Sphagna of Australia and Tasmania. Proc. Linn. Soc. NSW 37: 383-389.
Watts, W.W. \& Whitelegge, H.L.K., 1902: Census muscorum Australiensium. Proc. Linn. Soc. NSW suppl. 27: 1-90.

Watts, W.W. \& Whitelegge, H.L.K., 1906: Census muscorum Australiensium. Proc. Linn. Soc. NSW suppl. 30: 91-163.

WELCH, W.H., 1970: Hookeriaceae species and distribution in Africa, Europe, Asia, Australia and Oceania. Proc. Indiana Acad Sci. 79: 377-387.

Weymouth, W.A., 1894: Some additions to the moss flora of Tasmania. Pap. Proc. R. Soc. Tasm. (1893): 200210.

WEYMouTh, W.A., 1896: Some additions to the moss flora of Tasmania. Part II. Pap. Proc. R. Soc. Tasm. (1895): $106-120$.

Weymouth, W.A., 1903: Some additions to the bryological flora of Tasmania. III. Pap. Proc. R. Soc. Tasm. (1902): 115-132.

WeYMouTh, W.A. \& Rodway, L., 1922: Bryophyte notes. Pap. Proc. R. Soc. Tasm. (1921): 173-175.

Willis, J.H., 1950: The chequered story of two Tasmanian mosses. Vict. Nat. 67: 30-35.

WILLIS, J.H., 1952. Systematic notes on Victorian nıosses 1. Vict. Nat. 69: 15--18.

WILlis, J.H., 1953: Nemataceae, a moss family new to Australia. Nature 172: 127-128.

WILLIS, J.H., 1955a. Systematic notes on Victorian mosses 4. Vict. Nat. $71: 157-163$.

WILLIS, J.H., 1955b: New and interesting moss records for Australia. Vict. Nat. 72: 73-78.

WILLIS, J.H., 1957: New records of mosses for Australian states. Vict. Nat. 74; 101-105.

WILSON, W., 1859; Musci. In Hooker, J.D. (Ed.): THE BOTANY OF THE ANTARCTIC VOYAGE, PART 3. FLORA TASMANIAE. VOL. 2. L. Reeve and Co, London: 160-221.

Yamaguchi, T., Seppelt, R.D., Iwatsuki, Z. \& Buchanan, A.M., 1990: Sphagnum (sect. Buchanania) leucobryoides sect. et sp. nov. from Tasmania. J. Bryol. 16: 45-54.

\section{(accepted 10 August 1990)}

P.J. Dalton

Department of Plant Science, University of Tasmania, GPO Box 252C, Hobart, Tasmania, Australia 7001

R.D. Seppelt

Antarctic Division, Channel Highway, Kingston, Tasmania, Australia 7050

A.M. Buchanan

Tasmanian Herbarium, GPO Box 252C, Hobart, Tasmania, Australia 7001 